\title{
An experimental approach for the determination of axial and flexural wavenumbers in circular exponentially tapered bars
}

\author{
Michał K. Kalkowski*, Jen M. Muggleton, Emiliano Rustighi \\ University of Southampton, Highfield, Southampton SO17 1BJ, UK
}

\begin{abstract}
Whilst the dynamics of tapered structures have been extensively studied numerically and analytically, very few experimental results have been presented to date. The main aim of this paper is to derive and demonstrate an experimental method enabling both axial and flexural wavenumbers in exponentially tapered bars to be estimated. Our particular interest in this type of tapering is motivated by its occurrence in naturally grown structures such as tree roots, with an outlook towards remote root mapping. Decomposing a dynamic response into a sum of contributing waves, we propose a method in which two independent wavenumbers can be calculated from five equispaced measurements. The approach was demonstrated in an experiment on a freely suspended wooden specimen supported by theoretical modelling. For axial waves we used the well-established elementary rod theory, whereas for flexural waves we build a piecewise uniform model based on the Timoshenko beam theory. The estimates calculated from the experimental data were compared with the analytical and numerical results and showed good agreement. The limitations of the method include an appropriate choice of sensor spacing, the effect of sensor misalignments and the assumption of small wavenumber variation for flexural waves.
\end{abstract}

Keywords: tapered rods, tapered beams, wave propagation, wavenumber estimation, tree root mapping, piecewise uniform

\section{Introduction}

Tapered bars or ducts can be found in many areas of industry. Wave propagation in such structures has received considerable previous attention, with the classical acoustic horn $[1,2]$ as the foremost ex4 ample. Depending on the type of tapering, waves undergo phenomena specific to that class of structure,

\footnotetext{
*Corresponding author

Email address: M.Kalkowski@s oton.ac.uk (Michał K. Kalkowski)
} 
5

6

7

8

such as frequency-banded energy transmission [3] or the acoustic black hole effect [4-6]. Tapered structures most often propagate energy without internal reflections as the local changes of the properties are not abrupt.

Whilst wave propagation in tapered bars defined in an industrial context is the most common research motivation, certain types of tapering are observed in naturally grown structures. For instance, it is known that tree roots' diameter decreases exponentially in the vicinity of the stem, a region commonly referred to as the 'zone of rapid taper' [7]. In recent years there has been an increased interest in exploration and maintenance of the underground space, especially in urban environments. In this context, non-destructive methods capable of interrogating buried components using on-surface measurements are of primary interest. Building on the successful application of vibroacoustic methods for locating buried water pipes [8], similar principles are expected to support remote tree root detection. To realise the latter, a better understanding of wave propagation in root structures and how they radiate into the surrounding soil is required.

Stress wave propagation in tree roots is, to our best knowledge, not well documented in the literature. However, given the exponential variation of the root's radius, the fundamental characteristics of wave propagation are expected to be similar to those of typical isotropic examples available in the literature. Numerous previous studies on the dynamics of tapered bars address specifically the topic of vibration of a finite system; this is, of course, directly related to wave propagation. The investigations into axial waves in tapered rods belong to one family of problems together with the aforementioned acoustic horn, or a duct with a variable cross-section, since the governing equations have the same form $[1,9,10]$.

Waves in tapered rods have been treated with a multitude of mathematical approaches. Stevenson [2] used the WKB (Wentzel, Kramers, Brillouin) approximation to formulate solutions for horns of an arbitrary shape enabling the identification of transmission and attenuation zones. Nayfeh and Telionis [9] employed the multiple scales method to study wave propagation in ducts with a varying cross-section. For specific tapering configurations, axial wave propagation and vibration were described using Bessel functions [11, 12]. Finally, Langley [13] performed an extensive investigation into wave propagation in non-homogeneous waveguides (including both axial and flexural motion) with the aid of perturbation methods.

Exponential tapering, which is of interest in this paper, allows for an analytical solution, if the elementary rod theory is used. Nevertheless, more complex rod theories may well be addressed by the 
aforementioned methods. One other possible approach is to formulate the problem in a transfer matrix manner [14]. Based on that concept, Gan et al. [3] presented a study comparing different rod theories for non-uniform rods focusing on pass-bands and cut-off frequencies.

A similar range of mathematical approaches have been applied to flexural vibration of exponentially tapered beams. Bessel functions were used for certain types of polynomial variation of the cross-sectional properties by Cranch and Adler [15]. Following their work, numerous researchers studied various configurations of linearly tapered beams, e.g. $[16,17]$. Some forms of a polynomial variation were addressed by Abrate [18] in a study concerning both axial and flexural waves. Suppiger and Taleb [19] showed that an exponentially tapered beam can be solved using simple exponential functions, but only for certain configurations not including a circular cross-section. To circumvent the limitations on the type of tapering that allows for an analytical solution, several numerical approaches have been adopted (see e.g the comparison in [20]). For instance, stiffness and mass matrices pertaining to finite element procedures for certain types of tapering have been calculated by Gupta [21] and Banerjee and Williams $[22]$.

Exponential tapering of circular bars, which is of interest in this paper, is understood as an exponential variation of the radius of the beam. Analytical solution for such variation does not exist, regardless of whether the Euler-Bernoulli or the Timoshenko beam theory is adopted. Apart from the conventional finite element procedures, an exponentially tapered circular bar can be solved using the analytical wave approach in which the dynamics of the structure are written in terms of propagating waves [23, 24]. Khoshbayani-Arani et al. [25] used this technique to investigate free vibration of rods and beams with a variable cross-section. They approximated a smooth variation of the structure's properties with a set of uniform segments which in the present paper is referred to as the piecewise uniform model. More recently, Nielsen and Sorokin [26] used the WKB approximation for analysis of wave propagation in curved beams of a slowly varying diameter.

The literature on the topic, of which only a fraction was invoked above, clearly indicates that multiple mathematical treatments of the title problem have been successfully developed and facilitated gaining a deep insight into the physics of wave propagation in tapered structures. However, to our best knowledge, apart from the notable work by Petersson and Numan [27], no experimental results on wave propagation in tapered bars have been published.

In the present paper we propose a new methodology allowing for an experimental investigation of wave propagation in exponentially tapered rods and beams in which the wavenumbers are estimated 
from equally-spaced frequency response function (FRF) measurements. Motivated by the tree root problem, we demonstrate the concept by estimating both axial and flexural wavenumbers in a wooden exponentially tapered circular bar from measured data. Given the expected frequency range of interest for tree root detection, we confined our attention to fundamental waves only. The experimental results are compared to the established theoretical solutions.

Whilst the treatment of axial waves is simple from a mathematical viewpoint, flexural waves in this type of structure cannot be solved analytically. Instead we formulated a piecewise uniform model that allows for calculating the desired response in a semi-analytical manner. The experimental approach adopted in this paper informs future work on wave radiation from exponentially tapered bars into the surrounding medium. Moreover, some aspects of wave measurements on such structures are discussed.

The paper is organised as follows. In Section 2 , we briefly present the analytical model for exponentially tapered rod and derive the equations for the estimation of the wavenumbers from five measured dynamic responses. Next, we introduce the experimental setup and discuss the results gathered for axial wave propagation comparing them with the analytical prediction. In Section 3 we focus on flexural waves. Firstly, the piecewise uniform model is briefly described together with calculation of the imaginary part of the wavenumber from the WKB approximation. Then, we present the experimental flexural wavenumber estimates at a few locations and discuss observed features. The paper is concluded in Section 4 with some general comments and implications for future work.

\section{Axial wave propagation}

Axial wave propagation in exponentially tapered structures is analysed first. We used the elementary rod theory that assumes a uniform distribution of the displacement over the cross-section and neglects the effects of Poisson's contraction. The displacement is taken time-harmonic and the $\mathrm{e}^{\mathrm{j} \omega t}$ term (where $\omega$ is the angular frequency and $t$ is time) is omitted hereafter for clarity. The equation of motion in the frequency domain is [10]

$$
U^{\prime \prime}(x)+\frac{A^{\prime}(x)}{A(x)} U^{\prime}(x)+\omega^{2} \frac{\rho}{E} U(x)=0
$$

where $U(x)$ is the complex amplitude of the displacement, $A(x)$ is the cross-sectional area of the rod, $\rho$ is the density, $E$ is the Young's modulus and the prime denotes differentiation over the propagation direction, $x$. A hysteretic or structural damping model is used to represent energy loss in the material 
throughout this paper; the Young's modulus is complex and expressed as $E=\bar{E}(1+\jmath \eta)$ where $\bar{E}$ is the storage modulus and $\eta$ is the loss factor. Other material damping models can be used alternatively.

Noting that the exponential tapering yields an $x$-dependence of the radius $r$

$$
r(x)=R_{0} \mathrm{e}^{-\beta x}
$$

where $R_{0}$ is the radius at the large face and $\beta$ is the flare constant, the governing equation can be written in a simpler form

$$
U^{\prime \prime}(x)-2 \beta U^{\prime}(x)+\omega^{2} \frac{\rho}{E} U(x)=0
$$

\subsection{Analytical solution for free waves}

The type of tapering considered in this paper results in Eq. (3) having constant coefficients, which allows for an analytical solution. Assuming that $U(x)=U \mathrm{e}^{-\mathrm{j} k x}$ we obtain

$$
-k^{2}+\mathrm{j} 2 \beta k+\omega^{2} \frac{\rho}{E}=0
$$

from which the wavenumbers are calculated as

$$
k=\mathrm{j} \beta \mp \sqrt{-\beta^{2}+k_{n}^{2}}
$$

In the above equation $k_{n}^{2}$ denotes the square of the uniform rod wavenumber $\left(\omega^{2} \frac{\rho}{E}\right)$.

A brief inspection of Eq. (5) indicates that there exists a cut-off frequency $\left(\omega=\beta c_{L}\right.$, where $c_{L}=$ $\sqrt{\operatorname{Re}(E) / \rho}$ is the rod wavespeed) below which both wavenumbers are imaginary, i.e. the associated waves are evanescent. Above the cut-off frequency there are two propagating waves, negative- and positive-going. The amplitude of the positive-going wave (in the direction of diminishing radius) grows, whereas the amplitude of the negative-going wave decreases, as indicated by the imaginary part of the wavenumber. At high frequencies the real part of the wavenumber of the tapered rod approaches the uniform rod solution.

In Fig. 1 we plot the wavenumber dispersion curves and attenuation for a circular exponentially tapered $\operatorname{rod}\left(E=10 \times(1+0.005 \mathrm{j}) \mathrm{GPa}, \rho=700 \mathrm{~kg} / \mathrm{m}^{3}, \beta=1.55, R_{0}=0.1 \mathrm{~m}\right)$. The attenuation defines the rate of decay of the wave and is derived from the imaginary part of the wavenumber

$$
\text { attenuation }=-20 \log (\mathrm{e}) \operatorname{Im}(k) R_{0}
$$


(a)

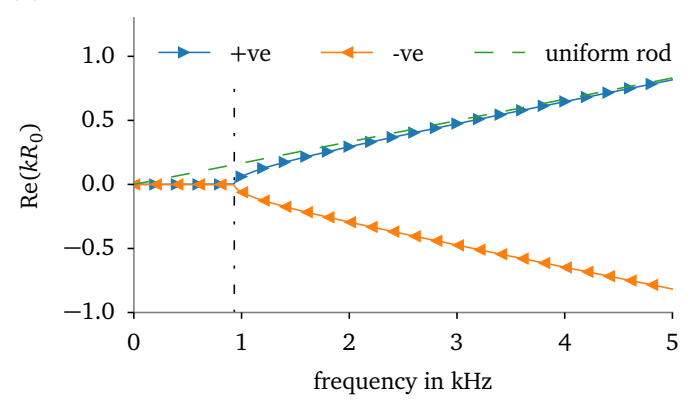

(b)

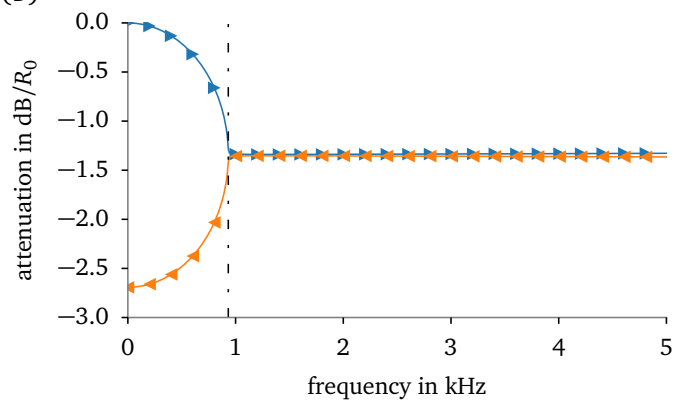

Figure 1: Dispersion curves for an exponentially tapered $\operatorname{rod}\left(E=10 \times(1+0.005 \mathrm{j}) \mathrm{GPa}, \rho=700 \mathrm{~kg} / \mathrm{m}^{3}, \beta=1.55, R_{0}=0.1 \mathrm{~m}\right)$ : (a) real part of the wavenumber; (b) attenuation.

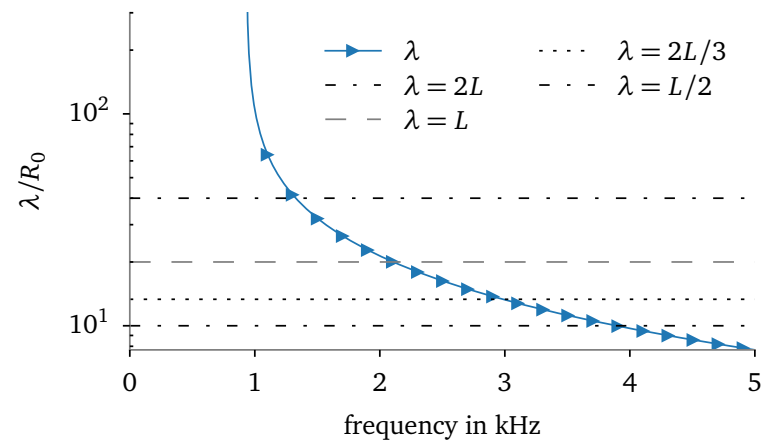

Figure 2: Axial wavelength in the exponentially tapered $\operatorname{rod}\left(E=10 \times(1+0.005 \mathrm{j}) \mathrm{GPa}, \rho=700 \mathrm{~kg} / \mathrm{m}^{3}, \beta=1.55, R_{0}=0.1 \mathrm{~m}\right)$ against frequency; lines corresponding to certain number of wavelengths within the length of the rod are also marked. 


\subsection{Axial wavenumber estimation}

The primary focus of this paper is to facilitate an experimental investigation of the wavenumbers in exponentially tapered structures. One possible approach was presented by Muggleton et al. [28] who estimated wavenumbers in a pipe using dynamic responses taken at three equispaced locations. Their method originated from the observation that the steady-state response is a superposition of waves travelling across the structure. As only one wave type was expected to be propagating in that case and $k^{+}=-k^{-}$, simple relationships were derived.

Based on a similar principle, Denis et al. [29] derived a method for estimating the reflection coefficient of an acoustic black hole termination. In their approach, a far-field assumption allowed for calculating propagating waves amplitudes from a series of measured dynamic responses. There is no particular requirement on the distribution of the measurement points, provided that their locations are known. The method, however, relies upon the a priori knowledge of the wavenumber in the beam.

The estimation of unknown wavenumbers is of primary interest here, hence the same starting point as in [28] is adopted. For an exponentially tapered rod the positive- and negative-going wavenumbers are different, i.e. $k^{+} \neq-k^{-}$. Consequently, the response can be written as a function of two unrelated wavenumbers

$$
u(x, t)=u_{1} \mathrm{e}^{\mathrm{j}\left(\omega t-k_{1} x\right)}+u_{2} \mathrm{e}^{\mathrm{j}\left(\omega t-k_{2} x\right)}
$$

where $k_{1}$ and $k_{2}$ are the two wavenumbers and $u_{1}$ and $u_{2}$ are the associated wave amplitudes. Note that no attribution of the direction of propagation is made at this stage.

The number of required sensing location comes from the number of unknowns (wavenumbers and wave amplitudes) and the form of Eq. (7). Muggleton et al. [28] showed that three sensing locations suffice if there is only one wave type propagating and $k^{+}=-k^{-}$. In the case presented here there are four unknowns in total. However, we found that a convenient manipulation of a set of sums of exponential terms can be done if five sensing locations are used (resulting in five simultaneous equations). Dropping the time-harmonic term and denoting the spacing between the sensors as $L_{s}$ five equispaced displacements $u_{a \ldots e}$ can be written as 


$$
\begin{aligned}
& u_{a}=u_{1} \mathrm{e}^{-\mathrm{j} k_{1} x_{0}}+u_{2} \mathrm{e}^{-\mathrm{j} k_{2} x_{0}} \\
& u_{b}=u_{1} \mathrm{e}^{-\mathrm{j} k_{1}\left(x_{0}+L_{s}\right)}+u_{2} \mathrm{e}^{-\mathrm{j} k_{2}\left(x_{0}+L_{s}\right)} \\
& u_{c}=u_{1} \mathrm{e}^{-\mathrm{j} k_{1}\left(x_{0}+2 L_{s}\right)}+u_{2} \mathrm{e}^{-\mathrm{j} k_{2}\left(x_{0}+2 L_{s}\right)} \\
& u_{d}=u_{1} \mathrm{e}^{-\mathrm{j} k_{1}\left(x_{0}-L_{s}\right)}+u_{2} \mathrm{e}^{-\mathrm{j} k_{2}\left(x_{0}-L_{s}\right)} \\
& u_{e}=u_{1} \mathrm{e}^{-\mathrm{j} k_{1}\left(x_{0}-2 L_{s}\right)}+u_{2} \mathrm{e}^{-\mathrm{j} k_{2}\left(x_{0}-2 L_{s}\right)}
\end{aligned}
$$

148

where $x_{0}$ is the location of the central sensor with respect to a chosen origin. A series of arithmetic manipulations of the above equations leads to the following set of simultaneous equations

$$
\begin{aligned}
\mathrm{e}^{\mathrm{j} k_{1} L_{s}}+\mathrm{e}^{\mathrm{j} k_{2} L_{s}} & =C_{1} \\
\mathrm{e}^{-\mathrm{j} k_{1} L_{s}}+\mathrm{e}^{-\mathrm{j} k_{2} L_{s}} & =C_{2}
\end{aligned}
$$

where $C_{1}=\frac{u_{b} u_{e}-u_{a} u_{d}}{u_{b} u_{d}-u_{a}^{2}}$ and $C_{2}=\frac{u_{d} u_{c}-u_{a} u_{b}}{u_{b} u_{d}-u_{a}^{2}}$.

Eq. (9) can be solved by the substitution

$$
\mathrm{e}^{\mathrm{j} k_{2} L_{s}}=\frac{1}{C_{2}-\mathrm{e}^{-\mathrm{j} k_{1} L_{s}}}
$$

which leads to

$$
C_{2} \mathrm{e}^{2 \mathrm{j} k_{1} L_{s}}-C_{1} C_{2} \mathrm{e}^{\mathrm{j} k_{1} L_{s}}+C_{1}=0
$$

Eq. (11) is an ordinary quadratic equation, so

$$
\mathrm{e}^{\mathrm{j} k_{1} L_{s}}=\frac{C_{1} C_{2} \pm \sqrt{C_{1} C_{2}\left(C_{1} C_{2}-4\right)}}{2 C_{2}}
$$

Finally, the wavenumber is obtained from

$$
k_{1}=\frac{1}{\mathrm{j} L_{s}} \ln \left(\frac{C_{1} C_{2} \pm \sqrt{C_{1} C_{2}\left(C_{1} C_{2}-4\right)}}{2 C_{2}}\right)
$$

The right hand side of Eq. (12) is a complex number, so a complex definition of logarithm must be used while evaluating $k_{1}$ in Eq. (13). The other wavenumber is obtained from the substitution equation, yet the analysis of the form of Eq. (9) indicates that the pairs of solutions for $k_{1}$ and $k_{2}$ are identical. Hence, it is enough to solve the above equation for $k_{1}$ which then captures both wavenumbers. 


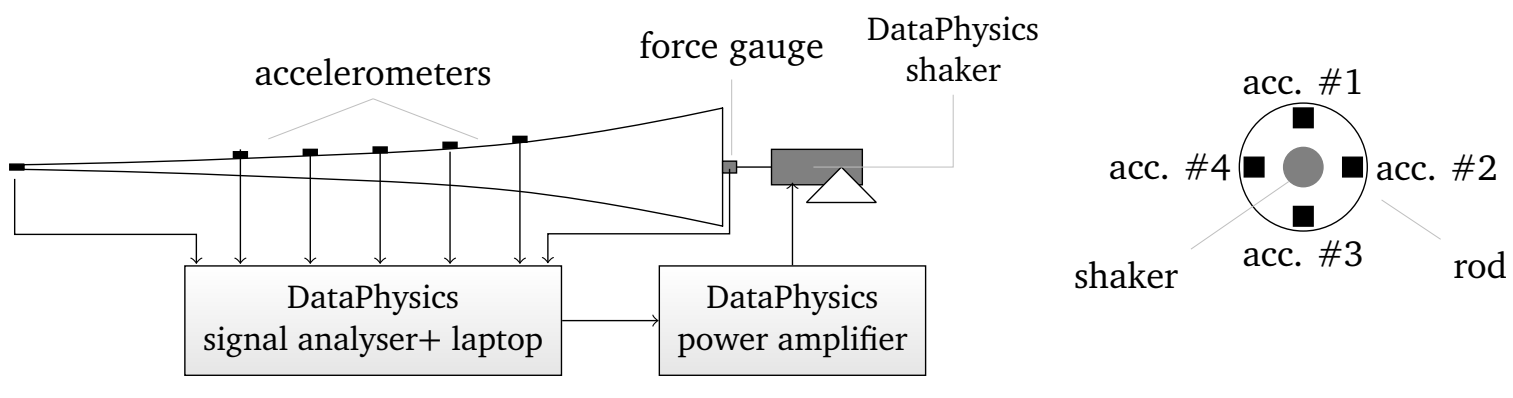

Figure 3: Experimental setup for axial FRF measurement: (a) schematic diagram; (b) accelerometers arrangement (planar view of the large face)

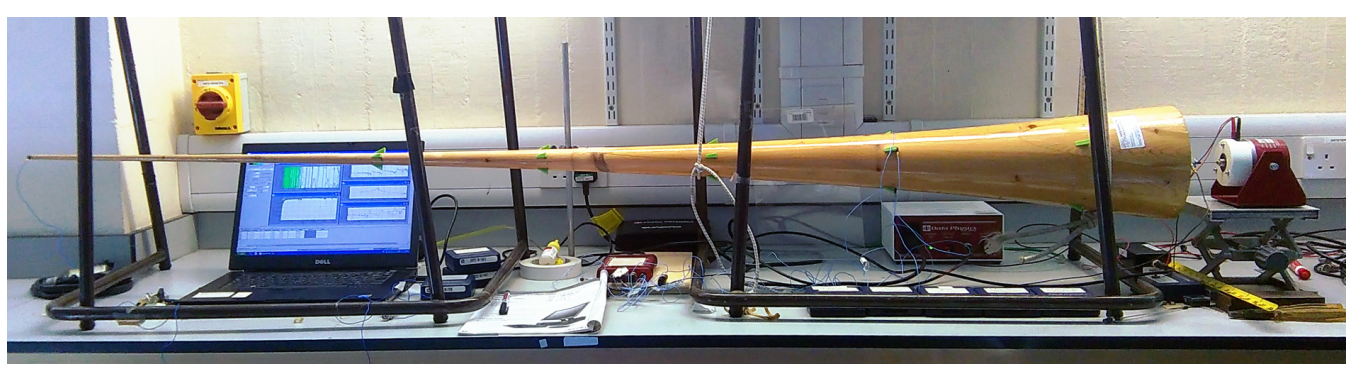

Figure 4: A photograph of the experimental setup.

\subsection{Experiment}

We performed an experiment on a freely suspended wooden rod with an exponentially varying radius. The rod was $2.007 \mathrm{~m}$ long and its radius varied in an exponential manner starting at from 0.0945 $\mathrm{m}$. To circumvent some of the manufacturing difficulties, the rod was not strictly homogeneous as it was a glued assembly of a few smaller parts. This, together with the anisotropy of the material not captured by the model presented in the previous sections, was expected to introduce some deviations from the predictions. However, as the elementary rod theory allows uniform axial deformation only, an effective Young's modulus can be determined which describes well the elastic properties of the specimen over a given frequency range. Moreover, given the uncertainties and relatively large tolerances in the manufacturing process of the specimen, we decided to identify the value for the flare constant $\beta$ from the measurements, so that the impact of the imperfections is minimised.

The experimental setup is schematically depicted in Fig. 3(a) and shown on a photograph in Fig. 4. The rod was excited with an electrodynamic shaker at the end with larger radius and instrumented with 
PCB 35C22 accelerometers. The input force was measured by a PCB208C01 force gauge connected to a shaker via a stinger. At each sensing location we used four accelerometers evenly distributed around the circumference as shown in Fig. 3(b). This enabled the influence of flexural waves, which were excited due to a non-ideally axial forcing and material anisotropy, to be eliminated from the measurements by simple arithmetic manipulation of the obtained FRFs. At each frequency, a mean of the four complex numbers was calculated which, thanks to the location of the sensors, retained only the contribution of the wave associated with uniform, in-phase motion of the cross-section plane. The accelerometers were mounted on the surface of the rod using 3D-printed attachments to facilitate orienting them so that predominantly axial response was captured.

\subsubsection{Material properties updating - point and transfer FRFs}

The effective properties of the rod were estimated based on measured point and transfer FRFs. The updating was performed using a simple least-squares minimisation of a cost function based on the real and imaginary parts of the simulated and measured responses.

The results for the point and transfer FRFs are shown in Fig. 5, where the predicted results are computed using the updated properties $\left(E=13.89 \times(1+0.0073 \mathrm{j}) \mathrm{GPa}, \rho=531 \mathrm{~kg} / \mathrm{m}^{3}, \beta=1.643\right)$. There are three resonances visible which correspond to when an integer number of a half-wavelength matches the length of the rod. The imprints of flexural resonances, the presence of which was alluded to above, can be observed in the transfer FRF (Fig. 5) below $2 \mathrm{kHz}$.

The results show very good agreement except for the antiresonances in the point FRF. We expect the discrepancy to be related to an undesired bending wave excitation and the effect of associated nearfields which are not accounted for in the analytical model for axial waves.

\subsubsection{Axial wavenumber estimation}

In this section we apply the method described in Sec. 2.2 to a set of responses measured experimentally at five equally spaced locations. The sensor spacing has a significant effect on the quality and, possibly, required processing of the estimates [28]. Whilst a too small spacing (compared with the wavelength) makes the inversion procedure very prone to noise, largely spaced sensors (more than half a wavelength apart) suffer from spatial aliasing, providing a wrapped estimate of the wavenumber. The method discussed in this paper requires some a priori knowledge of the expected wavelengths, so that the sensor spacing can be chosen accordingly. The longest wavelength in the specimen was expected to 
(a)

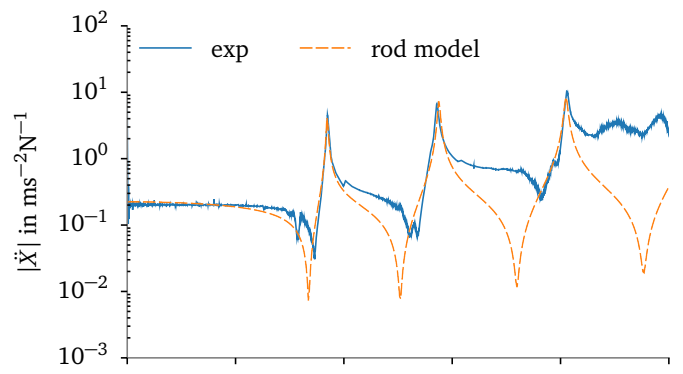

(c)

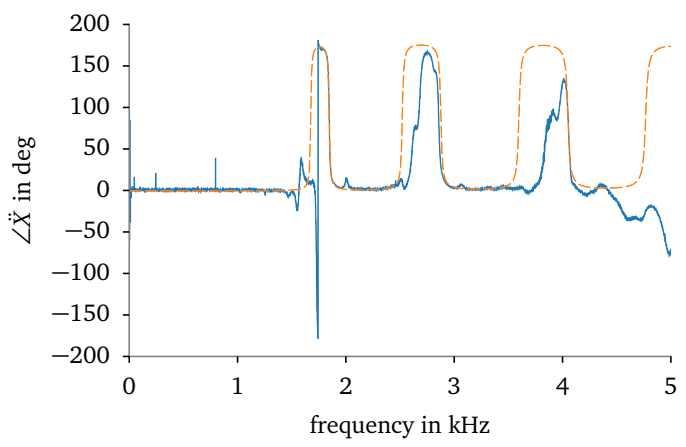

(b)

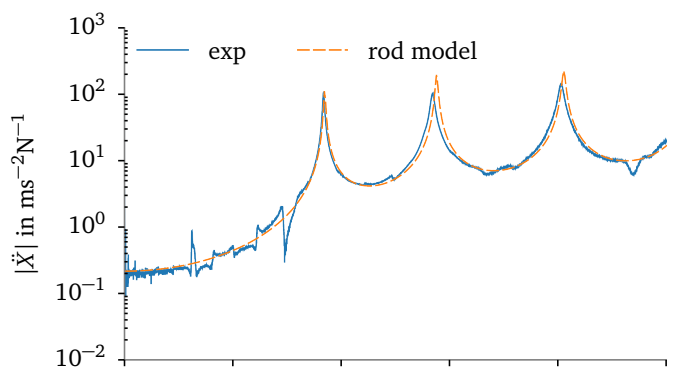

(d)

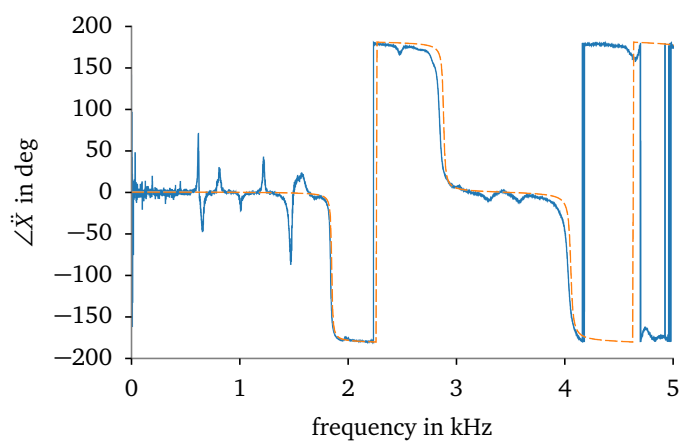

Figure 5: Comparison between the experimental and the analytical results: magnitude (a) and phase (c) of the point accelerance; magnitude (b) and phase (d) of the transfer accelerance. 
(a)

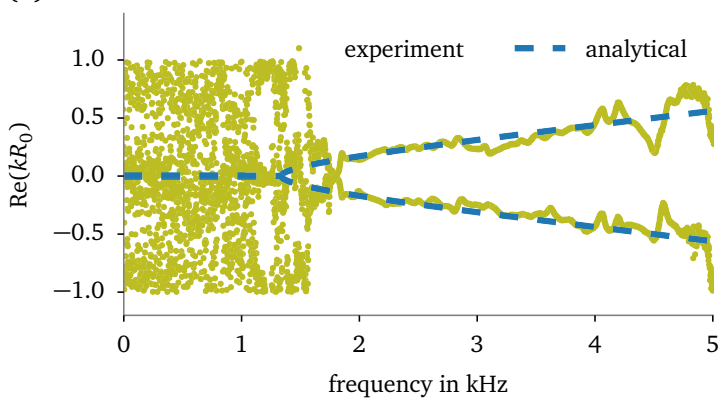

(b)

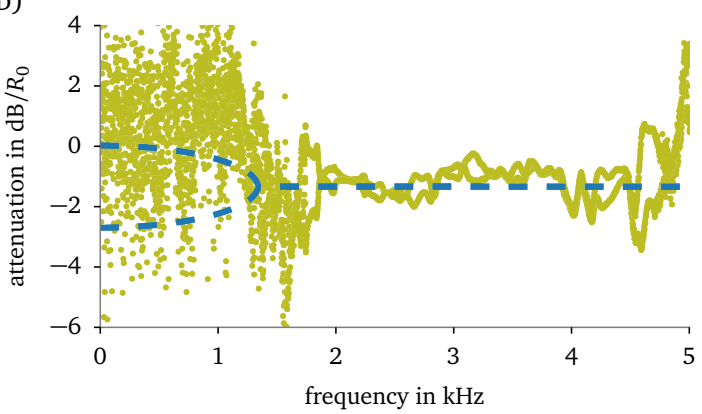

Figure 6: Axial wavenumber estimated from the experimental data (using the ring of four sensors at each location): (a) real part; (b) attenuation.

be $0.99 \mathrm{~m}$ at $5 \mathrm{kHz}$, therefore the maximum sensor spacing with no spatial aliasing was $0.445 \mathrm{~m}$. For this experiment, the sensors were spaced at $0.3 \mathrm{~m}$ (starting sensor at $0.15 \mathrm{~m}$ from the shaker)

Wavenumber estimates from the experimental data are compared with the analytical result in Fig. 6. The estimates follow the analytical trend closely and the cut-off phenomenon is well predicted. The resonance imprints on the estimates (e.g around $4.5 \mathrm{kHz}$ ) are thought to be related to residual flexural motion contribution which appears consequently to material anisotropy and non-ideally axial forcing.

The results below the cut-off frequency deserve a further explanation. In this region the wavenumber is predominantly imaginary, hence the phase difference between the responses along the rod is very small. Consequently, the outcome of the arithmetic manipulation of the dynamic responses used to estimate wavenumbers is dominated by measurement noise and contains little useful physical information. Owing to the numerical implementation of the logarithm, the wavenumber is limited to $\left(-\pi L_{s}, \pi L_{s}\right)$ range ( $L_{s}$ being the sensor spacing) which explains the arrangement of points in Fig. 6 and also suggests that the wave is, indeed, cut off.

The results in the propagating region are promising and prove the potential of the method for measuring wavenumbers in exponentially tapered rods. However, predominantly imaginary wavenumbers cannot be recovered if signals are noisy. The significance of the sensor spacing is demonstrated in Fig. 7 where the results with $0.15 \mathrm{~m}$ spacing are shown. The estimates are strongly affected by noise and extracting physical information becomes difficult.

Measuring dispersion curves for exponential circular rods requires a priori estimation of the wavelengths for an informed choice of sensor spacing. This can be done using the uniform rod wavelength 
(a)

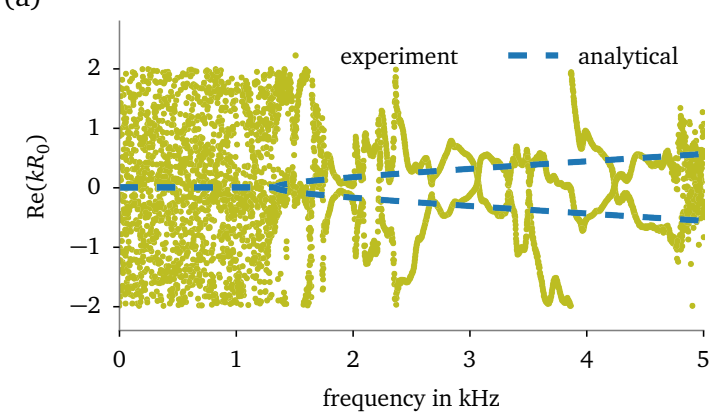

(b)

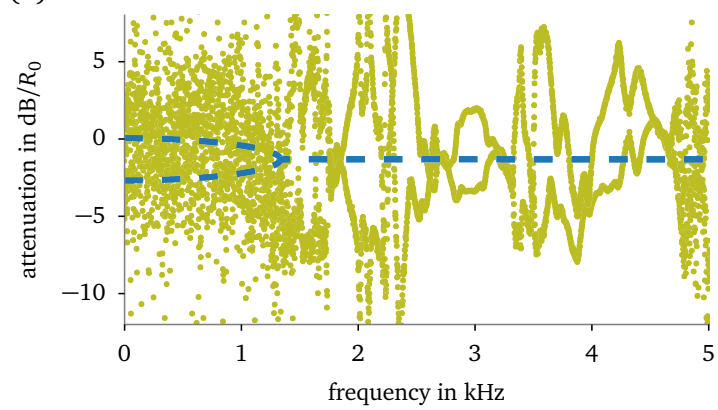

Figure 7: Axial wavenumber estimated from the experimental data - the effect of insufficient sensor spacing (here: $0.15 \mathrm{~m}$ ): (a) real part; (b) attenuation.

as at high frequencies the dispersion curve of the exponential rod tends to that of the uniform one. A spacing smaller than half of the shortest wavelength is preferred, although one needs to consider the noise level if the wavelengths are significantly larger than the spacing. It is also essential to pay attention to sensor alignment both with respect to the forcing direction and to the angular location along the circumference if the ring of sensors is used.

\section{Flexural wave propagation}

Flexural wave propagation in exponential beams is considered in this section. The analysis is based on the Timoshenko beam theory which accounts for the effects of shear deformation and rotary inertia, expected to play a role in structures of interest, especially near the large face. Corresponding governing equations are written as [10]

$$
\begin{aligned}
& \frac{\partial}{\partial x}\left[G A(x) \kappa\left(\psi-\frac{\partial w}{\partial x}\right)\right]+\rho A(x) \frac{\partial^{2} w}{\partial t^{2}}=q(x, t) \\
& G A(x) \kappa\left(\frac{\partial w}{\partial x}-\psi\right)+\frac{\partial}{\partial x}\left[E I(x) \frac{\partial \psi}{\partial x}\right]=\rho I(x) \frac{\partial^{2} \psi}{\partial t^{2}}
\end{aligned}
$$

where $G$ is the shear modulus, $\kappa$ is the Timoshenko shear coefficient, $y$ is the transverse displacement, $\psi$ is the rotation angle, $q(x, t)$ is the distributed load and $I(x)$ is the second moment of area. Note that both $A(x)$ and $I(x)$ are functions of $x$.

The tapering renders the coefficients of the above partial differential equations dependent on $x$. Unlike some types of tapering invoked in the literature review (e.g. $[15,18,19]$ ), an exponential 
variation of the radius does not allow for an analytical solution. Furthermore, a simple comparison with the uniform beam case, where the wavenumber is a function of frequency and the geometry of the cross-section (see Appendix A), indicates that the wavenumber depends now on the location along the beam:

$$
k(x)= \pm \sqrt{\frac{\omega^{2}}{2}\left(\frac{1}{C_{s}^{2}}+\frac{1}{C_{L}^{2}}\right) \mp \sqrt{\frac{\omega^{4}}{4}\left(\frac{1}{C_{s}^{2}}-\frac{1}{C_{L}^{2}}\right)^{2}+\frac{4 \omega^{2}}{C_{L}^{2}} R_{0}^{-2} \mathrm{e}^{2 \beta x}}}
$$

where

$$
C_{s}=\sqrt{\frac{G \kappa}{\rho}} \quad \text { and } \quad C_{L}=\sqrt{\frac{E}{\rho}}
$$

where complex $E$ and $G$ are used in the above expressions. Hence, flexural wave motion in circular exponential beams cannot be described by simple space-harmonic components.

One possible approach to solve Eq. (14) is to formulate it in a piecewise uniform manner. A tapered beam is discretised using uniform beam elements, under the assumption that the variation of the wavenumber in the tapered rod is small over the element length. The dynamics of the beam are written in terms of travelling waves that scatter at junctions between the neighbouring segments. Formulated in a matrix form, they can be easily implemented numerically, however a large number of elements may be needed for some cases to achieve desired accuracy.

Alternatively, one can use the WKB approximation, which enables the governing equation to be solved in the same way as it is commonly performed for uniform beams. In WKB the effect of the variation of the wavenumber along the beam is accounted for approximately using a phase integral. WKB approximation was not widely used for elastic wave propagation problems before a notable work by Pierce [30], as it carries an inherent contradiction between its short-wave nature and the assumptions of commonly used elastic theories pertaining to low frequencies. However, the overlap between the validity ranges of both WKB and Timoshenko beam theory were shown to be considerable [26]. Applications of the WKB approximation in structural vibration and acoustics are very wide and well known (see e.g. a review in [31]).

Although the method can work well for exponentially tapered circular beams, it is not fully implemented in this paper. The background application indicates that the greatest interest focuses on rather low frequencies for which the WKB is less effective. Nevertheless, it provides useful physical insight and simple mathematical treatment which is its great advantage. Hence, whilst most numerical simulations 
for flexural waves are performed using the piecewise uniform model in this paper, the WKB approximation is employed to obtain an analytical estimate for the evolution of the amplitude of the wave as a consequence of the tapering (imaginary part of the wavenumber).

The change of the amplitude between two locations is expressed as

$$
\gamma=\ln \frac{\tilde{W}\left(x_{1}\right)}{\tilde{W}\left(x_{0}\right)}
$$

where $\tilde{W}(x)$ is the WKB amplitude solution for Timoshenko waves derived by Pierce [30]

$$
\tilde{W}(x)=\sqrt{\frac{k(x)[1+\mu(x)]}{\rho A(x)}}
$$

where

$$
\mu(x)=\frac{I(x)}{2 A(x)}[E /(\kappa G)-1]\left[\omega^{2} \rho /(\kappa G)-k(x)^{2}\right]
$$

with $k$ being the Timoshenko wavenumber.

\subsection{Piecewise uniform model}

The key concepts related to the piecewise uniform model are outlined below. An exponentially tapered beam is divided into $n$ elements, over which the diameter does not vary significantly, so that they can be approximated as uniform. The dynamics of each element is written in terms of waves travelling across $\mathbf{a}_{i}^{+}, \mathbf{a}_{i}^{-}$(where $i$ is the element number) as depicted in Fig. 8. In this paper, the origins of waves are defined at locations where they enter the element, i.e. $\mathbf{a}_{i}^{+}$originate from the left boundary of the element, whereas $\mathbf{a}_{i}^{-}$from the right boundary. The phase and amplitude change along an element is described using a propagation matrix $\boldsymbol{\tau}_{i}$. There are $(n-1)$ junctions between the elements at which waves scatter, this being described by scattering matrices $\mathbf{T}^{(i)}$. Finally, matrices $\mathbf{R}_{L}, \mathbf{R}_{R}$ connect waves incident and reflected from the end boundaries. The beam is excited by a point force $Q$ acting on the left boundary and inducing a wave of amplitude $\mathbf{e}^{+}$.

Each element is modelled using a Timoshenko beam theory with cross-sectional properties evaluated according to the position. The detailed description of how the aforementioned matrices are calculated can readily be found in the literature (e.g. [32]) and are recalled in the Appendix A for completeness.

Most commonly, piecewise uniform models are solved using a transfer matrix-type approach, i.e. the global transfer matrix relating waves at the two ends of the structure is obtained by successive 


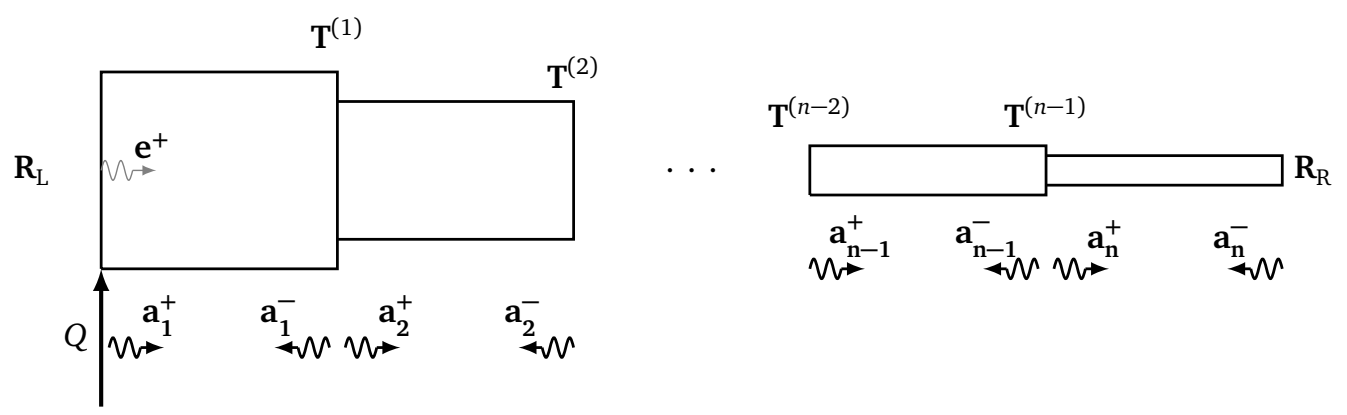

Figure 8: Piecewise uniform model - schematic representation of the wave description.

multiplications of element transfer matrices. However, this approach was found prone to numerical instabilities if a large number of elements was used and the variation of properties among the elements was relatively large. For exponentially tapered beams, the radii at the two ends are more than an order of magnitude different, hence the numerical problems were well observed in this case.

To circumvent these limitations we used an alternative approach in which the origins of all waves are defined at the point where they enter a wave element (see Fig. 8). This enabled the repeated multiplication of matrices with very small and very large elements to be avoided. Moreover, instead of being written in terms of a global transfer matrix, the system is now solved recursively. These two measures provided a robust solution method regardless of the number of elements or the distribution of properties assigned. Its detailed description is presented in Appendix B.

\subsection{Estimating flexural dispersion curves}

Flexural wavenumbers in tapered circular beams were estimated using the same framework as described in Section 2.2. Although the equations in Section 2.2 were derived for position-invariant wavenumbers, it is believed that they can give informative results for flexural motion as well, if a small variation of the wavenumber between the sensors is assumed. This, however, is in contradiction with the aforementioned observation indicating that the method works well only if the sensor spacing is sufficiently large so that the phase difference of the response between adjacent locations can be resolved in the presence of noise. A trade-off between these conditions exists and requires careful consideration for each specific case. 


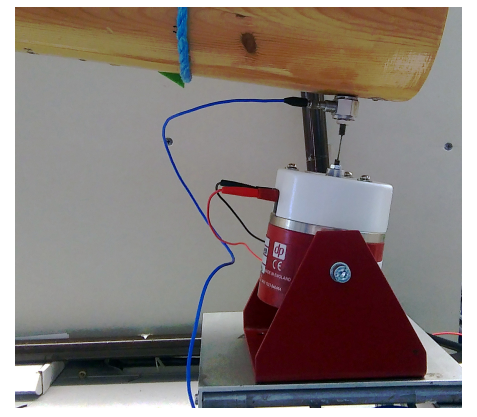

Figure 9: Shaker arrangement for flexural waves experiments.

\subsection{Experiment}

An experiment was performed on the same specimen as in Section 2.2. The shaker was reconfigured to excite predominantly flexural motion as shown in Fig. 9. To reduce the effect of axial wave contributions, two accelerometers mounted on the opposite sides of the beam were used to capture the response.

\subsubsection{Flexural frequency response functions}

Firstly, the point and transfer FRFs were recorded, and then used to establish the material properties. Since the material was not isotropic, the properties were identified independently of those in Section 2.3. Unlike for the axial waves, the anisotropy of the material was expected to play a significant role in this case. However, provided that the elastic to shear modulus ratio $(E / G)$ is specified appropriately, the Timoshenko equation developed for isotropic beams can yield accurate results as noted by Nowiński [33], who considered uniform orthotropic Timoshenko beams. In this paper, we followed Nowiński's approach using isotropic Timoshenko beam equations but specifying the elastic and shear moduli independently.

To identify the properties, we minimised the weighted sum of the natural frequency errors, over the first eleven natural frequencies, between the experiment and the numerical data. This resulted in the following properties: $E=15.66 \mathrm{GPa}, E / G=17.14$. The method used to establish the material properties is somewhat simplistic, but the main aim was to verify whether the isotropic model was capable of representing the fundamental physical phenomena in the real wooden specimen correctly. It is worth mentioning that these properties fall into a range of values typically observed for pine wood [34]. The loss factor for both elastic moduli was assumed to be 0.02 . The comparison between the 
(a)

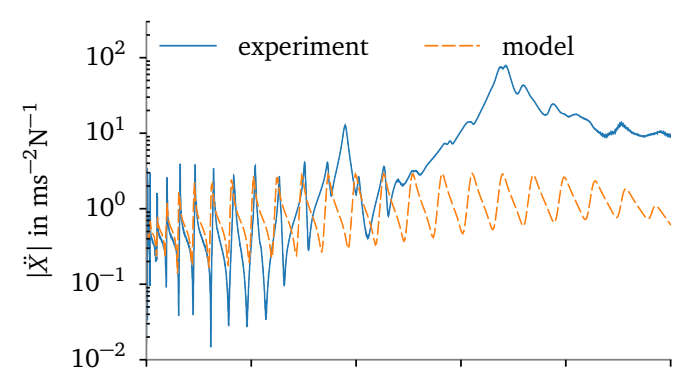

(c)

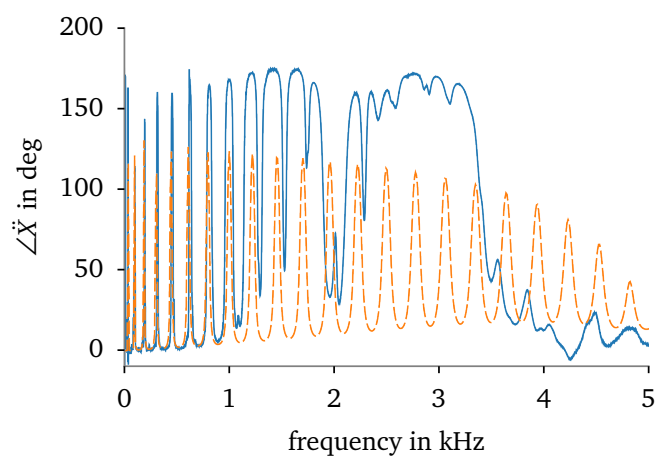

(b)

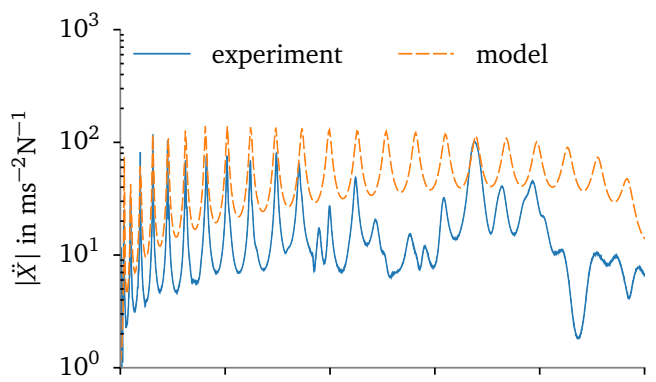

(d)

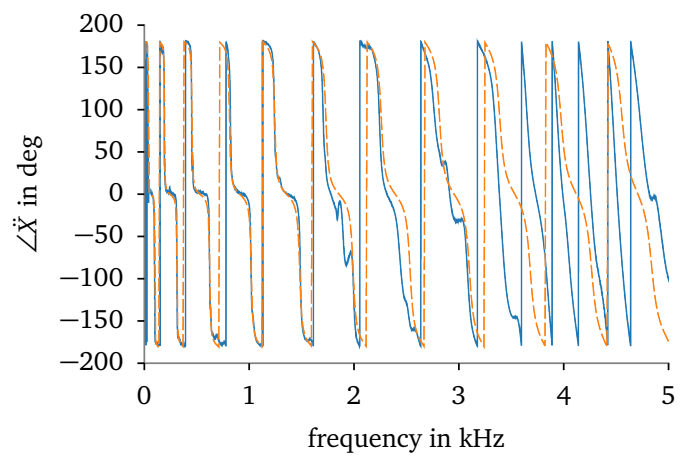

Figure 10: Comparison between the experimental and the numerical results: magnitude (a) and phase (c) of the point accelerance; magnitude (b) and phase (d) of the transfer accelerance.

measured data and simulated results based on these properties is shown in Fig. 10.

Fig. 10 indicates that the resonance frequencies are predicted correctly up to at least $2 \mathrm{kHz}$. The difference in magnitude is attributed to the fact that the experimental specimen is not deforming in the plane given by the force direction and beam axis only. Owing to the material anisotropy and possibly misaligned forcing, both the orthogonal bending and the axial waves are also excited. However, despite the aforementioned differences, the simulated results represent the fundamental physical characteristics of the real structure well, even though its material structure is much more complex than the isotropic configuration assumed in the model.

The measured response of the beam above $2 \mathrm{kHz}$ fails to follow the expected flexural behaviour. In this frequency range the response was found to be dominated by an ovalling wave that started propagating near the forcing location. Its associated cross-section deformation pattern is depicted schematically in Fig. 11 based on the measurement with four accelerometers around the circumference. The cut-off 


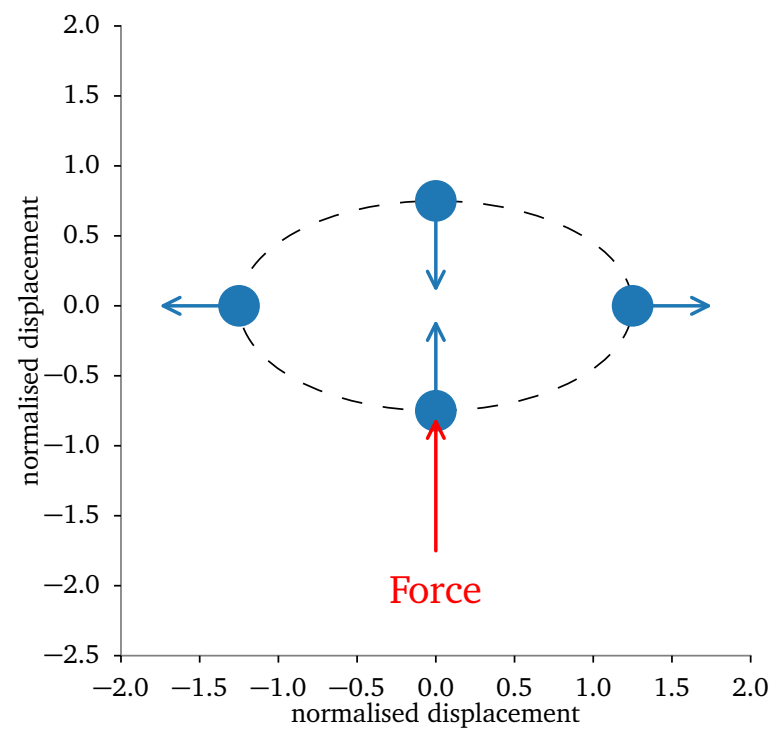

Figure 11: Cross-sectional view of the measured response (ODS) at $2430 \mathrm{kHz}$ dominated by the ovalling wave near the forcing location; dots represent the position of accelerometers and the arrows anchored at the dots - the displacement.

frequency of this wave depends on the radius of the cross-section, hence it varies with the position along the beam.

Although the higher-order waves not captured by the model are predominant in the response above $2 \mathrm{kHz}$, the results show that an appropriately formulated isotropic model can still describe well waves in exponentially tapered structures at low frequencies.

\subsubsection{Flexural wavenumber estimation}

In this section we estimate the flexural dispersion curves from both simulated and measured FRFs using the approach outlined in Section 2.2. As mentioned before, the application of this approach to flexural waves is associated with the assumption that the variation of the wavenumber between the sensors is small. Wavenumbers estimated in this way are thought to represent the wavenumber at the location of the central sensor.

To provide background for this calculation it is informative to look at the wavelength distribution along the beam at different frequencies. The wavelength distribution at a few representative frequencies calculated from Eq. (15) is shown in Fig. 12. A spacing of $0.075 \mathrm{~m}$ was chosen for the experiment, which is expected to give a wrapped wavenumber estimate for locations closer to the tip at high frequencies. 


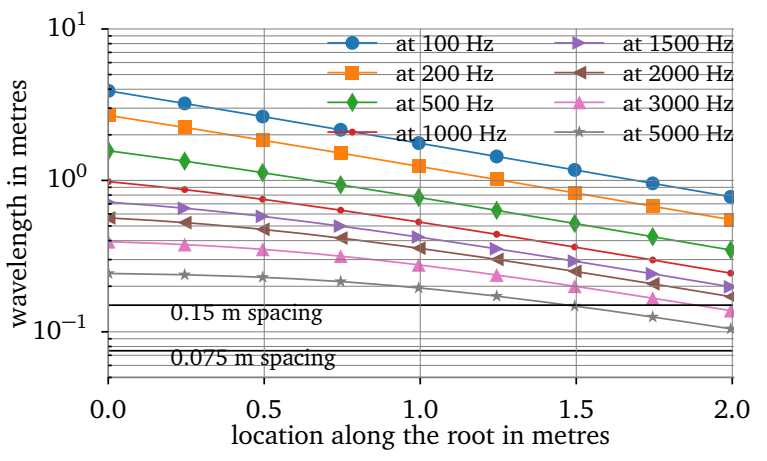

Figure 12: Flexural wavelength distribution along the beam at different frequencies with the main adopted spacing and its double marked.

Both the spacing and its double are marked in Fig. 12 for reference.

Wavenumbers were estimated at a few locations (of the central sensor), three of which are discussed below: $0.4 \mathrm{~m}, 1.05 \mathrm{~m}$ and $1.725 \mathrm{~m}$ from the large face with the spacing of $0.075 \mathrm{~m}$. At each of the sensing locations we used the data from two accelerometers placed in line with the force. This was expected to allow for cancelling the contributions of longitudinal waves and some higher-order waves, e.g. the ovalling wave mentioned before. The corresponding estimates of the wavenumbers are shown in Figs. 13-15. For reference, all figures contain a numerical estimate based on the responses calculated from the piecewise uniform model and a WKB-based prediction of the wave attenuation showing the evolution of the wave amplitude due to the tapering.

The estimate in Fig. 13 was computed from FRFs measured around the central sensor placed 0.4 $\mathrm{m}$ from the large face. The frequency range in which the response shows predominantly the flexural behaviour is the smallest near the large face. Consequently, the estimate at $0.4 \mathrm{~m}$ follows the analytical prediction for the central sensor up to around $3 \mathrm{kHz}$. Low frequencies are not resolved well, as the wavelength is long and only a small fraction is contained within sensor spacing. Measurement noise is amplified in the estimation procedure and overpowers physical information coming from the differences between adjacent FRFs. Above $3 \mathrm{kHz}$ the additional ovalling wave is expected to dominate the response. The fact that the wavenumber estimation routine was unable to extract flexural wave information indicates that the contributions of other waves were not cancelled by substituting the signals from two accelerometers mounted in line with the force, this being attributed to sensor misalignments. Nevertheless, the trend of the estimate below $3 \mathrm{kHz}$ agrees well with both the analytical and the numerical 
(a)

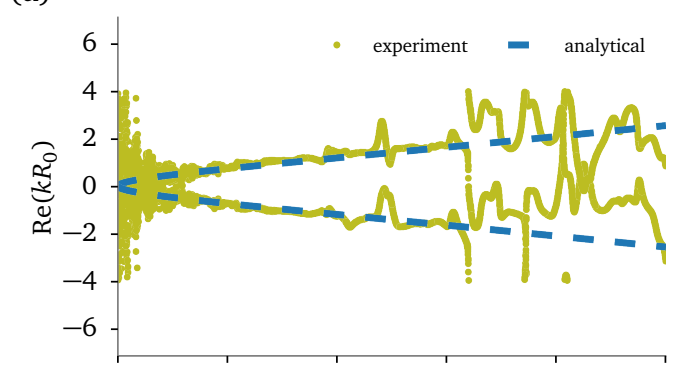

(c)

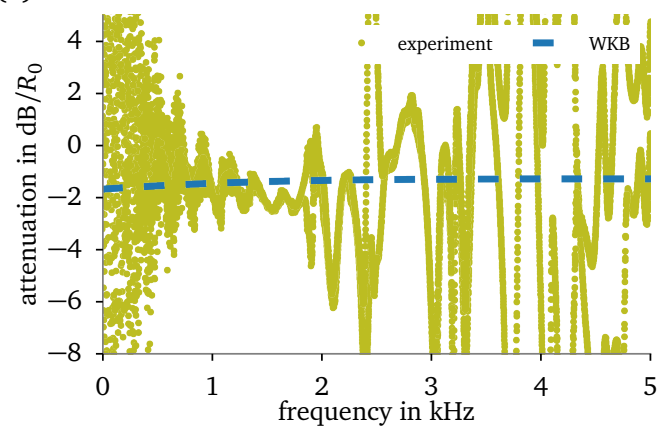

(b)

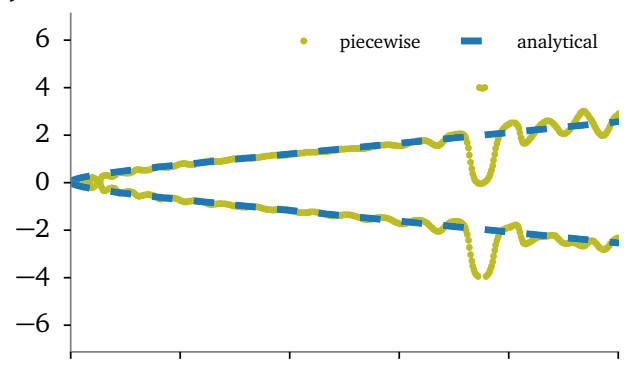

(d)

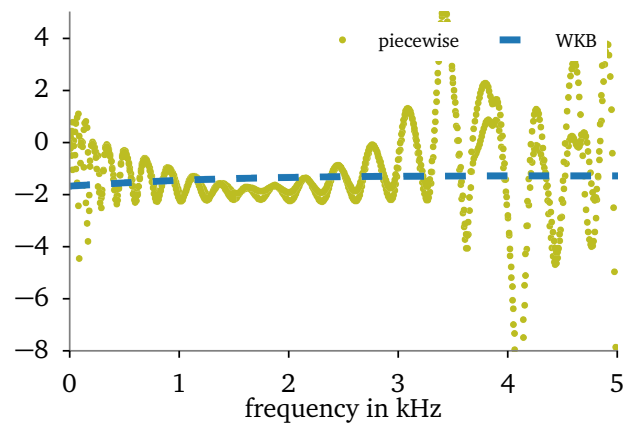

Figure 13: Flexural wavenumber estimated from the experimental and numerical data - five FRFs spaced at $0.075 \mathrm{~m}$ with the central sensor at $0.4 \mathrm{~m}$. From experimental FRFs: (a) real part; (c) attenuation. From numerical FRFs: (b) real part; (d) attenuation. 
(a)

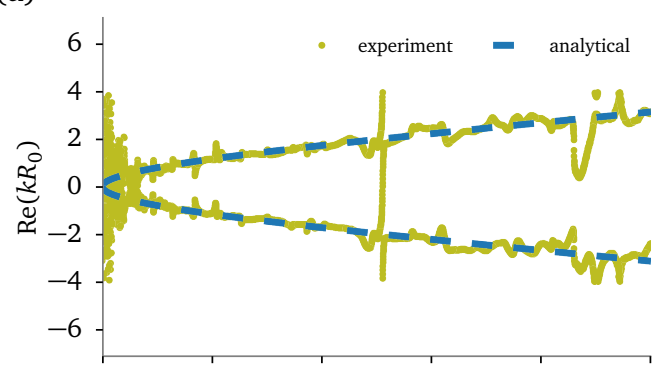

(c)

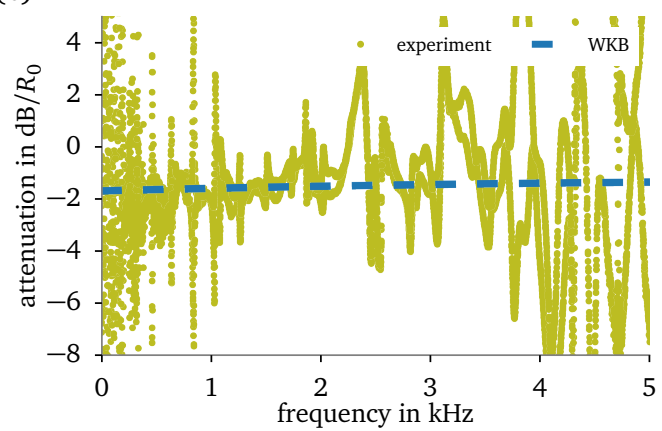

(b)

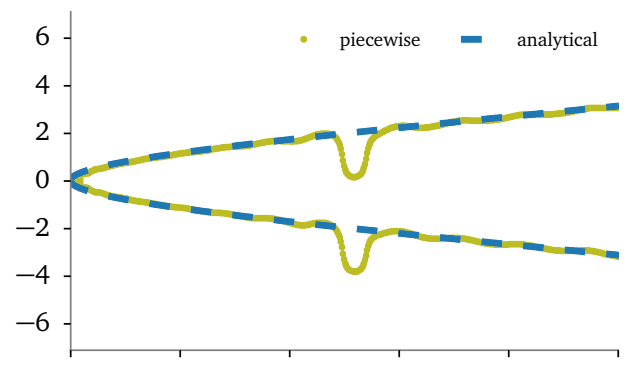

(d)

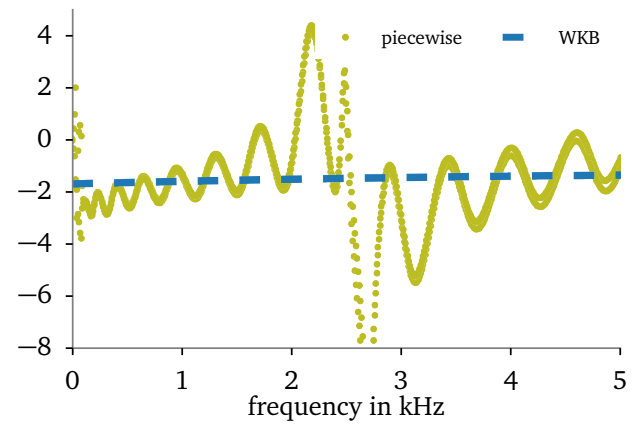

Figure 14: Flexural wavenumber estimated from the experimental and numerical data - five FRFs spaced at $0.075 \mathrm{~m}$ with the central sensor at 1.05 m. From experimental FRFs: (a) real part; (c) attenuation. From numerical FRFs: (b) real part; (d) attenuation.

prediction.

Further down the beam the wavenumber estimate improves, as shown in Fig. 14, where the central sensor is placed at $1.05 \mathrm{~m}$ from the large face. The estimate follows the analytical prediction over the whole frequency range presented. Between 2.5 and $3 \mathrm{kHz}$ there is a dip in the numerical results. It is attributed to the fact that the estimation routine assumes the wavenumber to be constant which is not, in fact, the case. However, we observed that decreasing the spacing in the numerical simulations moves the tip towards higher frequencies which indicated its association with sensor spacing and, in consequence, the wavenumber variation between the sensors.

The dip observed in the numerical results is less pronounced in the experimental data and located at slightly lower frequencies. Given that the characteristic features of the estimates in both numerical and experimental readings match, we infer that they are related to the wavenumber estimation procedure rather than to some physical features of the specimen. Both numerical and experimental results indicate 
(a)

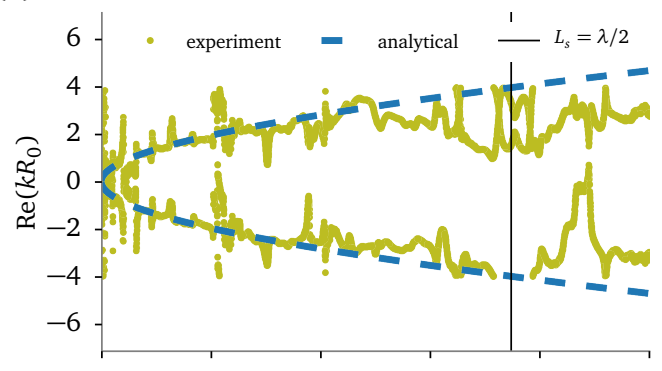

(c)

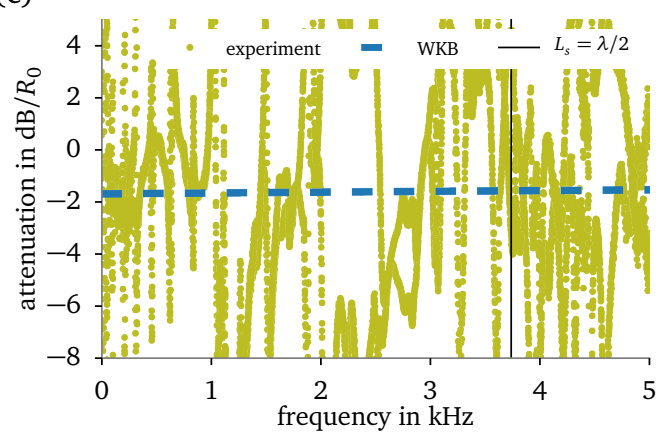

(b)

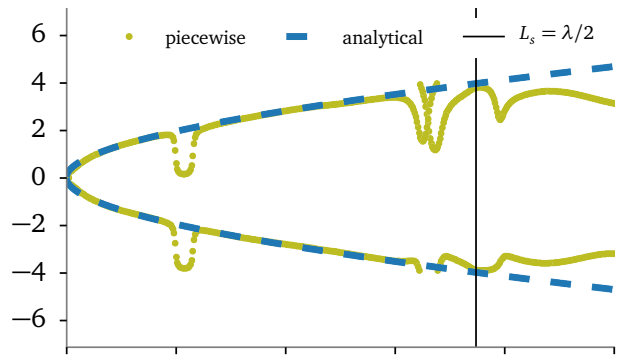

(d)

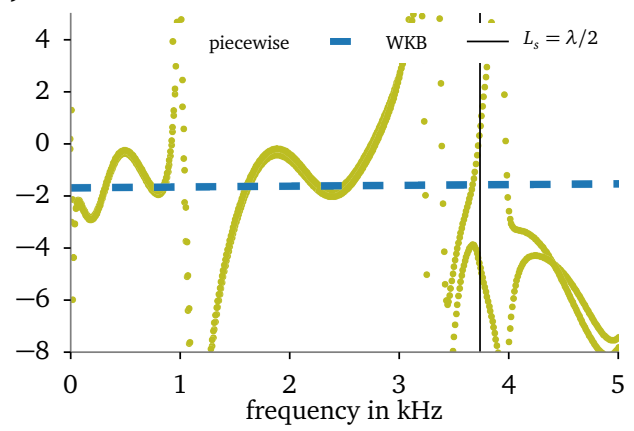

Figure 15: Flexural wavenumber estimated from the experimental and numerical data - five FRFs spaced at $0.075 \mathrm{~m}$ with the central sensor at 1.725 m. From experimental FRFs: (a) real part; (c) attenuation. From numerical FRFs: (b) real part; (d) attenuation.

that the imaginary part of the wavenumber is more sensitive to the effect of the wavenumber variation. Although the observed trend matches the theoretical prediction for wave amplitude evolution due to tapering, the oscillations around that line are very strong.

At the last considered location, which is relatively close to the tip of the beam (1.725 $\mathrm{m}$ from the large face), the effect of wavenumber wrapping is observed. The sensor spacing captures more than half-wavelength, hence spatial aliasing occurs. The frequency at which this condition is met is marked in Fig. 15. In addition, a small dip just above $1 \mathrm{kHz}$ (again, expected to be related to the wavenumber variation) is observed in both numerical and experimental results.

To asses the effect of the sensor spacing further, we took an additional measurement with the central sensor at $1.725 \mathrm{~m}$, but decreased spacing. The results for $0.04 \mathrm{~m}$ spacing are shown in Fig. 16. Although reduced spacing was expected to remove spatial aliasing, which was achieved in the numerical results, it introduced additional complications in the experiment. Firstly, the differences between the FRFs at 
(a)

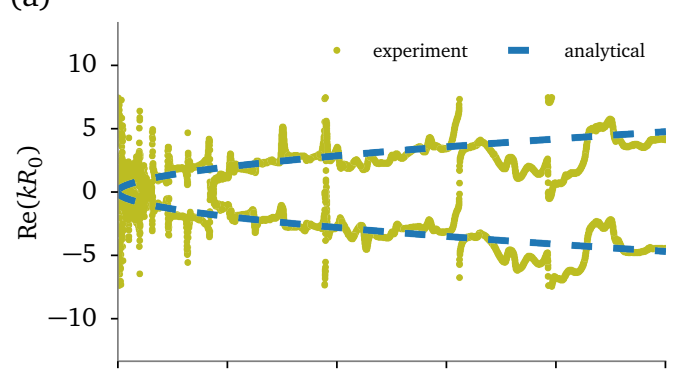

(c)

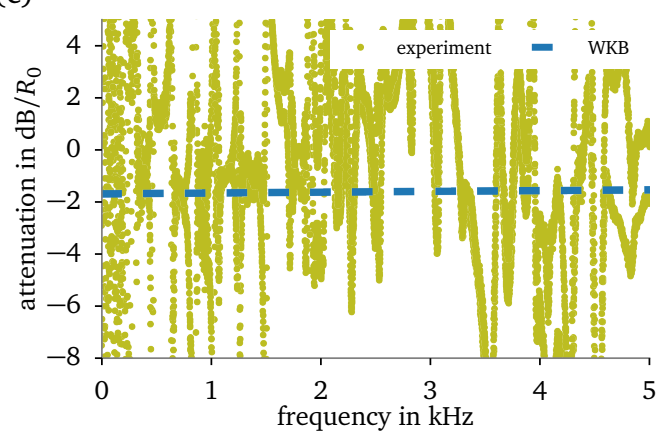

(b)

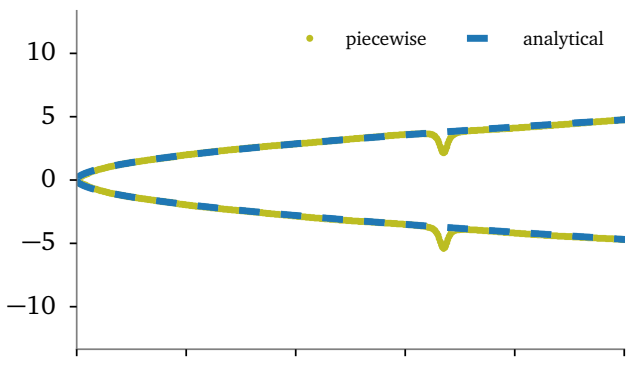

(d)

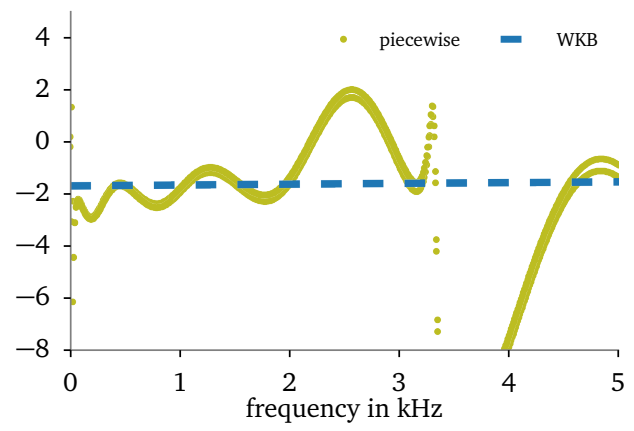

Figure 16: Flexural wavenumber estimated from the experimental and numerical data - five FRFs spaced at $0.04 \mathrm{~m}$ with the central sensor at 1.725 m. From experimental FRFs: (a) real part; (c) attenuation. From numerical FRFs: (b) real part; (d) attenuation.

considered locations became less significant relative to the noise. Secondly, sensor alignment imperfections started to play an important role as they become considerable with respect to sensor spacing. Moreover, aligning the sensors precisely with the forcing direction was quite difficult in the experimental setup. Finally, accelerometers have a finite area which, with decreasing spacing, constituted a significant proportion of that distance. All these factors affect the wavenumber estimates and a trade-off between them needs a careful consideration for each individual application.

Nevertheless, the examples discussed above indicate that the approach presented in this paper is capable of predicting flexural wavenumbers in tapered circular beams locally. Both wavenumber variation and wave evolution were shown to be position dependent following the trends from the analytical predictions. For measuring wavenumbers using the presented methodology, some prior knowledge of the possible range of values is required to inform the considerations of the effect of sensor spacing and the location along the beam. The effect of noise is significant since the estimation procedure involves a 
series of arithmetic operations on the measured FRFs. Therefore, the method might not work well for thick cross-sections (large wavelengths) as the response is usually very small there and signal-to-noise ratios may be poor for practical sensor spacings. Moreover, the alignment of sensors plays an important role, especially in the short wavelength region. Although using two sensors around the circumference should, in principle, support cancelling the contributions of other waves, it was not shown to be effective in the experiment, owing to sensor misalignments and complex dynamics of the specimen.

\section{Conclusions}

This paper considered axial and flexural waves in circular tapered structures focusing on facilitating wavenumber estimation from a small number of FRF measurements at equidistant locations.

Axial waves were modelled using elementary rod theory, which permits an analytical solution for exponential tapering. To capture both positive- and negative-going wavenumbers, a new estimation procedure was developed in which the dispersion curves were calculated from five equally spaced measurements. The experimental validation preceded by model parameters updating showed very good agreement with the theory. The effect of sensor spacing and the techniques used to cancel the contributions of other waves were also discussed.

The flexural wavenumber depends on the position along the beam, hence the dispersion curves estimates were calculated under the assumption of the wavenumber variation over the sensing region being relatively small. A set of illustrative results was presented indicating the trade-off between spatial aliasing, the resolvability of the wavenumbers from tightly spaced sensors and the aforementioned assumption of small wavenumber variation. Despite these restrictions, the technique was shown to provide satisfactory estimates for local wavenumbers which agreed well with the predictions. The estimation procedure developed in this paper can be applied to any structural configuration in which the two propagating waves are in general unrelated, such as any type of tapering.

In future work the influence of the surrounding medium on exponentially tapered circular bars embedded in infinite elastic medium will be considered.

\section{Acknowledgements}

The authors gratefully acknowledge the support provided by the EPSRC (under grant EP/K021699/1). The data supporting this study are openly available from the University of Southampton repository at http://dx.doi.org/10.5258/SOTON/394722. 


\section{Appendix A. Wave relationships for a uniform, homogeneous Timoshenko beam}

In this section we briefly recall some basic wave relationships for uniform beams originating from the Timoshenko theory, this being a basis upon which the piecewise uniform model presented in this paper was built. The governing equation for a homogeneous beam can be written as

$$
\begin{aligned}
G A \kappa\left(\frac{\partial \psi}{\partial x}-\frac{\partial^{2} w}{\partial x^{2}}\right)+\rho A \frac{\partial^{2} w}{\partial t^{2}} & =q(x, t) \\
G A \kappa\left(\frac{\partial w}{\partial x}-\psi\right)+E I \frac{\partial^{2} \psi}{\partial x^{2}} & =\rho I \frac{\partial^{2} \psi}{\partial t^{2}}
\end{aligned}
$$

where $G$ is the shear modulus, $\kappa$ is the Timoshenko shear coefficient, $w$ is the transverse displacement, $\psi$ is the rotation angle, $q(x, t)$ is the distributed load, $A$ is the cross-sectional area and $I$ is the crosssectional second moment of area.

Assuming the solutions to be space- and time-harmonic and omitting the time dependence, one writes the free waves equation in the matrix form as

$$
\left[\begin{array}{cc}
k^{2} G A \kappa-\rho A \omega^{2} & -\mathrm{j} k G A \kappa \\
-\mathrm{j} k G A \kappa & \rho I \omega^{2}-k^{2} E I-G A \kappa
\end{array}\right]\left\{\begin{array}{l}
W \\
\Psi
\end{array}\right\}=0
$$

which enables the relationship between the two degrees of freedom to be specified as

$$
\frac{\Psi}{Y}=-\mathrm{j} k\left(1-\frac{\omega^{2}}{k^{2} C_{s}^{2}}\right)=-\mathrm{j} P
$$

The dispersion equation comes from the non-vanishing determinant condition in Eq. (A.2) and is a biquadratic equation in $k$

$$
-k^{4}+k^{2} \omega^{2}\left(\frac{1}{C_{s}^{2}}+\frac{C_{r}^{2}}{C_{b}^{2}}\right)+\frac{\omega^{2}}{C_{b}^{2}}\left(1-\frac{\omega^{2} C_{r}^{2}}{C_{s}^{2}}\right)
$$

where

$$
C_{s}=\sqrt{\frac{G \kappa}{\rho}} \quad C_{b}=\sqrt{\frac{E I}{\rho A}} \quad C_{r}=\sqrt{\frac{I}{A}}
$$

from which the wavenumbers can be found

$$
k= \pm \sqrt{\frac{\omega^{2}}{2}\left(\frac{1}{C_{s}^{2}}+\frac{C_{r}^{2}}{C_{b}^{2}}\right) \mp \sqrt{\frac{\omega^{4}}{4}\left(\frac{1}{C_{s}^{2}}-\frac{C_{r}^{2}}{C_{b}^{2}}\right)^{2}+\frac{\omega^{2}}{C_{b}^{2}}}}
$$


Eq. (A.6) indicates that there are two pairs of wavenumbers pertaining to positive- and negative-going waves such that $\mathbf{k}^{+}=-\mathbf{k}^{-}$, where $\mathbf{k}^{+}=\left[\begin{array}{ll}k_{1}^{+} & k_{2}^{+}\end{array}\right]^{\top}$. Moreover, $\omega_{c}=\frac{C_{s}}{C_{r}}$ is a cut-off frequency, above which all wavenumbers are real (if no damping is present). Below $\omega_{c}$ one pair of solutions is real and the other imaginary. Each of the Timoshenko waves is associated with a wave mode shape given by the eigenvectors of Eq. (A.2) which then can be grouped with respect to the propagation direction

$$
\boldsymbol{\phi}_{q}^{+}=\left[\begin{array}{cc}
1 & 1 \\
-\mathrm{j} P\left(k_{1}\right) & -\mathrm{j} P\left(k_{2}\right)
\end{array}\right] \quad \text { and } \quad \boldsymbol{\phi}_{q}^{-}=\left[\begin{array}{cc}
1 & 1 \\
\mathrm{j} P\left(k_{1}\right) & \mathrm{j} P\left(k_{2}\right)
\end{array}\right]
$$

Thus, the response of the beam can be written in terms of travelling waves as

$$
\left\{\begin{array}{c}
Y \\
\Psi
\end{array}\right\}=\boldsymbol{\phi}_{q}^{+}\left\{\begin{array}{l}
a_{1}^{+} \\
a_{2}^{+}
\end{array}\right\}+\boldsymbol{\phi}_{q}^{+}\left\{\begin{array}{l}
a_{1}^{-} \\
a_{2}^{-}
\end{array}\right\}
$$

where $a_{1}, a_{2}$ correspond to the amplitude of the two Timoshenko waves. Likewise, it is often useful to write the resultant forces associated with particular waves in a similar wave denoting them as force mode shapes. Recalling that the resultant forces over the cross-section are defined as

$$
\begin{aligned}
& V=G A \kappa\left(\frac{\partial w}{\partial x}-\psi\right) \\
& M=E I \frac{\partial \psi}{\partial x}
\end{aligned}
$$

one writes the force mode shape matrices in terms of the displacement mode shape and its spatial derivative

$$
\boldsymbol{\phi}_{f}^{+}=\left[\begin{array}{cc}
0 & -G A \kappa \\
0 & 0
\end{array}\right] \boldsymbol{\phi}_{q}^{+}+\left[\begin{array}{cc}
G A \kappa & 0 \\
0 & E I
\end{array}\right] \frac{\mathrm{d} \boldsymbol{\phi}_{q}^{+}}{\mathrm{d} x}
$$

While waves propagate their amplitudes change according to the space-harmonic propagation matrix

$$
\tau(x)=\left[\begin{array}{ll}
\mathrm{e}^{-\mathrm{j} k_{1} x} & \\
& \mathrm{e}^{-\mathrm{j} k_{2} x}
\end{array}\right]
$$

where the direction of propagation is indicated by the sign of $x$.

At end boundaries waves undergo reflection and mode conversion which are described by reflection matrices. In this paper only free ends are considered, hence the boundary condition is that all resultant forces are zero 


$$
\boldsymbol{\Phi}_{f}^{+} \mathbf{a}^{+}+\boldsymbol{\Phi}_{f}^{-} \mathbf{a}^{-}=0
$$

Given that at the right boundary $\mathbf{a}^{-}=\mathbf{R}_{\text {free }} \mathbf{a}^{+}$, a reflection matrix can be calculated from the above equation

$$
\mathbf{R}_{\text {free }}=-\left[\boldsymbol{\Phi}_{\mathrm{f}}^{-}\right]^{-1} \boldsymbol{\Phi}^{+}
$$

Substituting the matrices and performing the calculation leads to

$$
\mathbf{R}_{\text {free }}=-\left[\begin{array}{cc}
-\frac{P_{1} k_{1}\left(P_{2}-k_{2}\right)+P_{2} k_{2}\left(P_{1}-k_{1}\right)}{k_{2} P_{2}\left(P_{1}-k_{1}\right)+k_{1} P_{1}\left(k_{2}-P_{2}\right)} & -\frac{2 P_{2} k_{2}\left(P_{2}-k_{2}\right)}{k_{2} P_{2}\left(P_{1}-k_{1}\right)+k_{1} P_{1}\left(k_{2}-P_{2}\right)} \\
\frac{2 p_{1} k_{1}\left(P_{1}-k_{1}\right)}{k_{2} P_{2}\left(P_{1}-k_{1}\right)+k_{1} P_{1}\left(k_{2}-P_{2}\right)} & \frac{P_{1} k_{1}\left(P_{2}-k_{2}\right)+P_{2} k_{2}\left(P_{1}-k_{1}\right)}{k_{2} P_{2}\left(P_{1}-k_{1}\right)+k_{1} P_{1}\left(k_{2}-P_{2}\right)}
\end{array}\right]
$$

Note that the reflection matrix for the left boundary is the same.

Finally, the calculation of wave amplitudes induced by a force or a moment is presented. In this paper we deal with boundary excitation only, hence only positive-going waves are excited. The force equilibrium equation is written as

$$
\left[\begin{array}{cc}
-\mathrm{j} k_{1}+\mathrm{j} P\left(k_{1}\right) & -\mathrm{j} k_{2}+\mathrm{j} P\left(k_{2}\right) \\
-k_{1} P\left(k_{1}\right) & -k_{2} P\left(k_{2}\right)
\end{array}\right] \mathbf{a}^{+}=\left\{\begin{array}{c}
\frac{-Q}{G A \kappa} \\
-M / E I
\end{array}\right\}
$$

from where

$$
\mathbf{a}^{+}=\frac{1}{k_{2} P_{2}\left(P_{1}-k_{1}\right)+k_{1} P_{1}\left(k_{2}-P_{2}\right)}\left[\begin{array}{cc}
-k_{2} P_{2} & P_{2}-k_{2} \\
k_{1} P_{1} & -P_{1}+k_{1}
\end{array}\right]\left\{\begin{array}{c}
\frac{-\mathrm{j} Q}{G A \kappa} \\
\frac{-M}{E I}
\end{array}\right\}
$$

\section{Appendix B. Piecewise uniform model (recursive formulation)}

In this section a recursive piecewise uniform formulation is presented. This approach was found stable regardless of the number of wave elements and variation of properties among the element. The more common and simpler formulation known as the transfer matrix approach may be prone to numerical instabilities for such configurations. The definition of the origins of propagating waves in an element is the key to stability. In this formulation the origins of the positive- and negative-going waves $\mathbf{a}^{+}, \mathbf{a}^{-}$ are always at the left and the right borders of its 'parent' wave elements, respectively. 


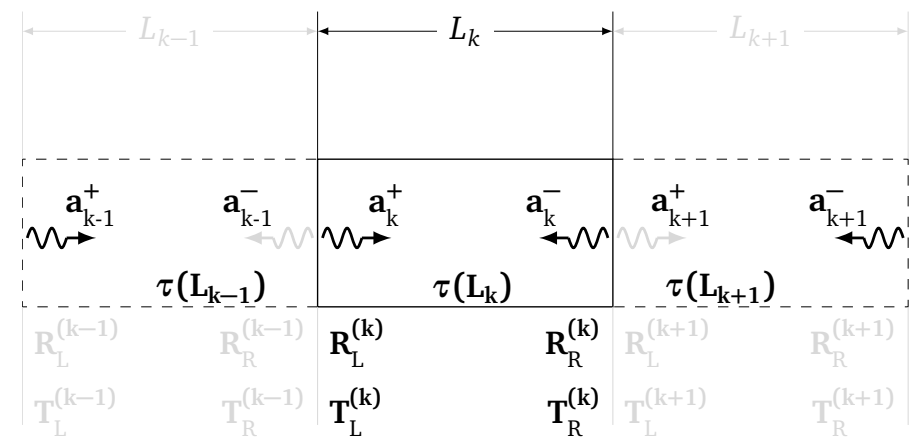

Figure B.1: Wave amplitudes and matrices required to fully describe the motion in a wave element.

Each junction between the elements involves wave scattering. Scattering matrix is calculated based on the approach presented in [23].

The waves required to fully describe wave motion in a wave element are shown in Fig. B.1. Matrices T, $\mathbf{R}, \boldsymbol{\tau}$ describe the scattering, reflection and propagation, respectively. Both positive- and negativegoing wave in an element can be described as a following superposition:

$$
\begin{aligned}
& \mathbf{a}_{k}^{+}=\mathbf{R}_{k}^{\mathrm{L}} \boldsymbol{\tau}_{k} \mathbf{a}_{k}^{-}+\mathbf{T}_{k}^{\mathrm{L}} \boldsymbol{\tau}_{(k-1)} \mathbf{a}_{(k-1)}^{+} \\
& \mathbf{a}_{k}^{-}=\mathbf{R}_{k}^{\mathrm{R}} \boldsymbol{\tau}_{k} \mathbf{a}_{k}^{+}+\mathbf{T}_{k}^{\mathrm{R}} \boldsymbol{\tau}_{(k+1)} \mathbf{a}_{(k+1)}^{-}
\end{aligned}
$$

The second equation can be substituted into the first yielding:

$$
\begin{aligned}
\mathbf{a}_{k}^{+} & =\mathbf{R}_{k}^{\mathrm{L}} \boldsymbol{\tau}_{k}\left[\mathbf{R}_{k}^{\mathrm{R}} \boldsymbol{\tau}_{k} \mathbf{a}_{k}^{+}+\mathbf{T}_{k}^{\mathrm{R}} \boldsymbol{\tau}_{(k+1)} \mathbf{a}_{(k+1)}^{-}\right]+\mathbf{T}_{k}^{\mathrm{L}} \boldsymbol{\tau}_{(k-1)} \mathbf{a}_{(k-1)}^{+} \\
& =\mathbf{R}_{k}^{\mathrm{L}} \boldsymbol{\tau}_{k} \mathbf{R}_{k}^{\mathrm{R}} \boldsymbol{\tau}_{k} \mathbf{a}_{k}^{+}+\mathbf{R}_{k}^{\mathrm{L}} \boldsymbol{\tau}_{k} \mathbf{T}_{k}^{\mathrm{R}} \boldsymbol{\tau}_{(k+1)} \mathbf{a}_{(k+1)}^{-}+\mathbf{T}_{k}^{\mathrm{L}} \boldsymbol{\tau}_{(k-1)} \mathbf{a}_{(k-1)}^{+}
\end{aligned}
$$

From where the piece-wise expression for the positive travelling wave is obtained:

$$
\mathbf{a}_{k}^{+}=\left[\mathbf{I}-\mathbf{R}_{k}^{\mathrm{L}} \boldsymbol{\tau}_{k} \mathbf{R}_{k}^{\mathrm{R}} \boldsymbol{\tau}_{k}\right]^{-1}\left[\mathbf{T}_{k}^{\mathrm{L}} \boldsymbol{\tau}_{(k-1)} \mathbf{a}_{(k-1)}^{+}+\mathbf{R}_{k}^{\mathrm{L}} \boldsymbol{\tau}_{k} \mathbf{T}_{k}^{\mathrm{R}} \boldsymbol{\tau}_{(k+1)} \mathbf{a}_{(k+1)}^{-}\right]
$$

Eq. (B.3) describes how the positive travelling wave in the element depends on the excited wave amplitudes and travelling waves in adjacent elements. To simplify the notation the following are adopted:

$$
\begin{aligned}
\mathbf{A}_{k}^{+} & =\left[\mathbf{I}-\mathbf{R}_{k}^{\mathrm{L}} \boldsymbol{\tau}_{k} \mathbf{R}_{k}^{\mathrm{R}} \boldsymbol{\tau}_{k}\right]^{-1} \\
\mathbf{C}_{k}^{+} & =\mathbf{A}_{k}^{+} \mathbf{T}_{k}^{\mathrm{L}} \boldsymbol{\tau}_{(k-1)} \\
\mathbf{D}_{k}^{+} & =\mathbf{A}_{k}^{+} \mathbf{R}_{k}^{\mathrm{L}} \boldsymbol{\tau}_{k} \mathbf{T}_{k}^{\mathrm{R}} \boldsymbol{\tau}_{(k+1)}
\end{aligned}
$$


An analogous procedure can be performed for negative travelling wave, in which case the coefficient matrices change slightly:

$$
\begin{aligned}
\mathbf{B}_{k}^{-} & =\left[\mathbf{I}-\mathbf{R}_{k}^{\mathrm{R}} \boldsymbol{\tau}_{k} \mathbf{R}_{k}^{\mathrm{L}} \boldsymbol{\tau}_{k}\right]^{-1} \\
\mathbf{C}_{k}^{-} & =\mathbf{B}_{k}^{-} \mathbf{R}_{k}^{\mathrm{R}} \boldsymbol{\tau}_{k} \mathbf{T}_{k}^{\mathrm{L}} \boldsymbol{\tau}_{(k-1)} \\
\mathbf{D}_{k}^{-} & =\mathbf{B}_{k}^{-} \mathbf{T}_{k}^{\mathrm{R}} \boldsymbol{\tau}_{(k+1)}
\end{aligned}
$$

With the aid of Eqs (B.4) and (B.5) a recursive expression for travelling waves in an element is written as:

$$
\begin{aligned}
& \mathbf{a}_{k}^{+}=\mathbf{C}_{k}^{+} \mathbf{a}_{(k-1)}^{+}+\mathbf{D}_{k}^{+} \mathbf{a}_{(k+1)}^{-} \\
& \mathbf{a}_{k}^{-}=\mathbf{C}_{k}^{-} \mathbf{a}_{(k-1)}^{+}+\mathbf{D}_{k}^{-} \mathbf{a}_{(k+1)}^{-}
\end{aligned}
$$

The equations above relate the unknown travelling waves in an element to the travelling waves in adjacent elements and therefore can readily be used in the piece-wise solver. The piece-wise solution starts from the far-right $\left(n^{\text {th }}\right)$ element for which the right incoming wave does not exist. Eq. (B.6) simplifies to:

$$
\begin{aligned}
& \mathbf{a}_{n}^{+}=\mathbf{C}_{n}^{+} \mathbf{a}_{(n-1)}^{+} \\
& \mathbf{a}_{n}^{-}=\mathbf{C}_{n}^{-} \mathbf{a}_{(n-1)}^{+}
\end{aligned}
$$

Moreover it is possible to relate $\mathbf{a}_{n}^{-}$to $\mathbf{a}_{n}^{+}$directly:

$$
\mathbf{a}_{n}^{-}=\mathbf{R}_{n}^{\mathrm{R}} \boldsymbol{\tau}_{n} \mathbf{a}_{n}^{+}
$$

Substituting Eq. (B.7) to Eq. (B.8) yields:

$$
\mathbf{a}_{n}^{-}=\mathbf{R}_{n}^{\mathrm{R}} \boldsymbol{\tau}_{n} \mathbf{C}_{n}^{+} \mathbf{a}_{(n-1)}^{+}=\mathbf{H}_{n}^{-} \mathbf{a}_{(n-1)}^{+}
$$

Matrix $\mathbf{H}_{\mathbf{n}}^{-}$represents how $\mathbf{a}_{(\mathbf{n}-1)}^{+}$contributes to the positive travelling wave in the wave element considered.

The general wave element equation can be now written for the $(n-1)^{\text {th }}$ element:

$$
\begin{aligned}
& \mathbf{a}_{(n-1)}^{+}=\mathbf{C}_{(n-1)}^{+} \mathbf{a}_{(n-2)}^{+}+\mathbf{D}_{(n-1)}^{+} \mathbf{a}_{n}^{-} \\
& \mathbf{a}_{(n-1)}^{-}=\mathbf{C}_{(n-1)}^{-} \mathbf{a}_{(n-2)}^{+}+\mathbf{D}_{(n-1)}^{-} \mathbf{a}_{n}^{-}
\end{aligned}
$$


Substituting Eq. (B.9) into Eq. (B.10) one gets:

$$
\begin{aligned}
& \mathbf{a}_{(n-1)}^{+}=\mathbf{C}_{(n-1)}^{+} \mathbf{a}_{(n-2)}^{+}+\mathbf{D}_{(n-1)}^{+} \mathbf{H}_{n}^{-} \mathbf{a}_{(n-1)}^{+} \\
& \mathbf{a}_{(n-1)}^{+}=\left[\mathbf{I}-\mathbf{D}_{(n-1)}^{+} \mathbf{H}_{n}^{-}\right]^{-1} \mathbf{C}_{(n-1)}^{+} \mathbf{a}_{(n-2)}^{+} \\
& \mathbf{a}_{(n-1)}^{+}=\mathbf{H}_{(n-1)}^{+} \mathbf{a}_{(n-2)}^{+}
\end{aligned}
$$

498

which provides an expression for the amplitude of the wave travelling in the wave element with respect to the positive travelling wave in the preceding section. Similarly, the recursive expression for the negative going wave can be established:

$$
\begin{aligned}
\mathbf{a}_{(n-1)}^{-} & =\mathbf{C}_{(n-1)}^{-} \mathbf{a}_{(n-2)}^{+}+\mathbf{D}_{(n-1)}^{-} \mathbf{a}_{n}^{-} \\
& =\mathbf{C}_{(n-1)}^{-} \mathbf{a}_{(n-2)}^{+}+\mathbf{D}_{(n-1)}^{-} \mathbf{H}_{n}^{-} \mathbf{a}_{(n-1)}^{+} \\
& =\mathbf{C}_{(n-1)}^{-} \mathbf{a}_{(n-2)}^{+}+\mathbf{D}_{(n-1)}^{-} \mathbf{H}_{n}^{-}+\mathbf{H}_{(n-1)}^{+} \mathbf{a}_{(n-2)}^{+} \\
& =\left(\mathbf{C}_{(n-1)}^{-}+\mathbf{D}_{(n-1)}^{-} \mathbf{H}_{n}^{-} \mathbf{H}_{(n-1)}^{+}\right) \mathbf{a}_{(n-2)}^{+} \\
& =\mathbf{H}_{(n-1)}^{-} \mathbf{a}_{(n-2)}^{+}
\end{aligned}
$$

The above equations can be applied to all internal wave elements. For the $1^{\text {th }}$ element, there is no incoming wave from the left side, hence the formulation is simplified to:

$$
\begin{aligned}
& \mathbf{a}_{1}^{+}=\mathbf{A}_{1}^{+} \mathbf{e}_{1}^{+}+\mathbf{D}_{1}^{+} \mathbf{a}_{2}^{-} \\
& \mathbf{a}_{1}^{-}=\mathbf{A}_{1}^{-} \mathbf{e}_{1}^{+}+\mathbf{D}_{1}^{-} \mathbf{a}_{2}^{-}
\end{aligned}
$$

Eq. (B.12) written for the $2^{\text {nd }}$ element can be then substituted back into Eq. (B.13):

$$
\begin{aligned}
\mathbf{a}_{1}^{+} & =\mathbf{A}_{1}^{+} \mathbf{e}_{1}^{+} \mathbf{D}_{1}^{+} \mathbf{H}_{2}^{-} \mathbf{a}_{1}^{+} \\
& =\mathbf{A}_{1}^{+} \mathbf{e}_{1}^{+}+\mathbf{D}_{1}^{+} \mathbf{H}_{2}^{-} \mathbf{a}_{1}^{+}
\end{aligned}
$$

From where one can solve for the positive travelling wave in the $1^{\text {st }}$ element:

$$
\mathbf{a}_{1}^{+}=\left[\mathbf{I}-\mathbf{D}_{1}^{+} \mathbf{H}_{2}^{-}\right]^{-1} \mathbf{A}_{1}^{+} \mathbf{e}_{1}^{+}
$$

After this solution is obtained travelling wave amplitudes in all wave elements are calculated on an element-by-element basis by substituting the left incoming travelling waves amplitudes into Eqs (B.11) and (B.12). 


\section{References}

[1] P. M. Morse, Vibration and Sound, McGraw-Hill Book Company Inc., New York, 1936.

[2] A. F. Stevenson, Exact and approximate equations for wave propagation in acoustic horns, Journal of Applied Physics 22 (12) (1951) 1461-1463. doi :10.1063/1.1699892.

[3] C. Gan, Y. Wei, S. Yang, Longitudinal wave propagation in a rod with variable cross-section, Journal of Sound and Vibration 333 (2) (2014) 434-445. doi:10.1016/j.jsv.2013.09.010.

[4] M. Mironov, Propagation of a flexural wave in a plate whose thickness decreases smoothly to zero in a finite interval, Soviet Physics - Acoustics 34 (1988) 318-319.

[5] V. Pisliakov, M. Mironov, A. Svadkovsky, Vibration of specially tapered beams and bars, in: Proceedings of the $29^{\text {th }}$ International Congress and Exhibition on Noise Control Engineering INTER NOISE, 27-30 August 2000, Nice, France, 2000, pp. 798-801.

[6] V. V. Krylov, F. J. B. S. Tilman, Acoustic 'black holes' for flexural waves as effective vibration dampers, Journal of Sound and Vibration 274 (3-5) (2004) 605-619. doi:10.1016/j.jsv.2003.05. 010.

[7] B. F. Wilson, The Growing Tree, University of Massachusetts Press, Amherst, 1984.

[8] J. M. Muggleton, M. J. Brennan, Y. Gao, Determining the location of buried plastic water pipes from measurements of ground surface vibration, Journal of Applied Geophysics 75 (1) (2011) 54-61. doi:10.1016/j.jappgeo.2011.06.030.

[9] A. H. Nayfeh, D. P. Telionis, Acoustic propagation in ducts with varying cross sections, The Journal of the Acoustical Society of America 54 (6) (1973) 1654-1661. doi:10.1121/1.1914464.

[10] K. Graff, Wave Motion in Elastic Solids, Dover Publications, Inc., New York, 1991.

[11] M. C. Junger, J. E. Cole III, Dispersion and cut-off phenomena in rods and beams, Tech. Rep. U-573-260, Cambridge Acoustical Associates (1978).

[12] B. M. Kumar, R. I. Sujith, Exact solutions for the longitudinal vibration of non-uniform rods, Journal of Sound and Vibration 207 (5) (1997) 721-729. doi:10.1006/jsvi.1997.1146. 
[13] R. S. Langley, Wave evolution, reflection, and transmission along inhomogeneous waveguides, Journal of Sound and Vibration 227 (1) (1999) 131-158. doi:10.1006/jsvi.1999.2337.

[14] M. Toso, Wave propagation in rods, shells, and rotating shafts with non-uniform geometry, $\mathrm{PhD}$, University of Maryland (2004).

[15] E. T. Cranch, A. A. Adler, Bending vibration of variable section beams, Journal of Applied Mechanics 23 (1) (1956) 103-108.

[16] H. H. Mabie, C. B. Rogers, Transverse vibrations of double-tapered cantilever beams, The Journal of the Acoustical Society of America 51 (5B) (1972) 1771-1774. doi :10.1121/1.1913028.

[17] R. P. Goel, Transverse vibrations of tapered beams, Journal of Sound and Vibration 47 (1) (1976) 1-7. doi : 10.1016/0022-460X (76) 90403-X.

[18] S. Abrate, Vibration of non-uniform rods and beams, Journal of Sound and Vibration 185 (4) (1995) 703-716. doi:10.1006/jsvi.1995.0410.

[19] E. W. Suppiger, N. J. Taleb, Free lateral vibration of beams of variable cross section, Zeitschrift für angewandte Mathematik und Physik ZAMP 7 (6) (1956) 501-520. doi:10.1007/BF01601179.

[20] P. A. A. Laura, R. H. Gutierrez, R. E. Rossi, Free vibrations of beams of bilinearly varying thickness, Ocean Engineering 23 (1) (1996) 1-6. doi:10.1016/0029-8018(95)00029-K.

[21] A. Gupta, Vibration of tapered beams, Journal of Structural Engineering 111 (1) (1985) 19-36. doi :10.1061/(ASCE) 0733-9445(1985) 111:1(19).

[22] J. R. Banerjee, F. W. Williams, Exact Bernoulli-Euler dynamic stiffness matrix for a range of tapered beams, International Journal for Numerical Methods in Engineering 21 (12) (1985) 2289-2302. doi:10.1002/nme.1620211212.

[23] N. R. Harland, B. R. Mace, R. W. Jones, Wave propagation, reflection and transmission in tunable fluid-filled beams, Journal of Sound and Vibration 241 (5) (2001) 735-754. doi:10.1006/ jsvi.2000.3316.

[24] S. K. Lee, B. R. Mace, M. J. Brennan, Wave propagation, reflection and transmission in non-uniform one-dimensional waveguides, Journal of Sound and Vibration 304 (1-2) (2007) 31-49. doi: $10.1016 / j \cdot j$ sv .2007.01.039. 
[25] M. Khoshbayani-Arani, N. Rasekh-Saleh, M. Nikkhah-Bahrami, A modified wave approach for calculation of natural frequencies and mode shapes in arbitrary non-uniform one-dimensional waveguides, Proceedings of the Institution of Mechanical Engineers, Part C: Journal of Mechanical Engineering Science 225 (12) (2011) 2864-2880. doi:10.1177/0954406211409804.

[26] R. Nielsen, S. Sorokin, The WKB approximation for analysis of wave propagation in curved rods of slowly varying diameter, Proceedings of the Royal Society A 470 (2167) (2014) 20130718. doi:10.1098/rspa.2013.0718.

[27] B. A. T. Petersson, E. J. M. Nijman, The one-dimensional bending wave counterpart to the acoustic horn and the semi-infinite wedge, Journal of Sound and Vibration 211 (1) (1998) 95-121. doi: 10.1006/jsvi.1997.1365.

[28] J. M. Muggleton, M. J. Brennan, P. W. Linford, Axisymmetric wave propagation in fluid-filled pipes: wavenumber measurements in in vacuo and buried pipes, Journal of Sound and Vibration 270 (1-2) (2004) 171-190. doi:10.1016/S0022-460X (03) 00489-9.

[29] V. Denis, F. Gautier, A. Pelat, J. Poittevin, Measurement and modelling of the reflection coefficient of an acoustic black hole termination, Journal of Sound and Vibration 349 (2015) 67 - 79. doi: http://dx.doi.org/10.1016/j.jsv.2015.03.043.

[30] A. D. Pierce, Physical interpretation of the WKB or eikonal approximation for waves and vibrations in inhomogeneous beams and plates, The Journal of the Acoustical Society of America 48 (1B) (1970) 275-284. doi:10.1121/1.1912125.

[31] A. T. Fabro, N. S. Ferguson, T. Jain, R. Halkyard, B. R. Mace, Wave propagation in one-dimensional waveguides with slowly varying random spatially correlated variability, Journal of Sound and Vibration 343 (2015) 20-48. doi:10.1016/j.jsv.2015.01.013.

[32] C. Mei, B. Mace, Wave reflection and transmission in timoshenko beams and wave analysis of timoshenko beam structures, Journal of Vibration and Acoustics 127 (4) (2005) 382-394. doi: $10.1115 / 1.1924647$.

[33] J. L. Nowiński, On the transverse wave propagation in orthotropic timoshenko bars, International Journal of Mechanical Sciences 11 (8) (1969) 689-693. doi :10.1016/0020-7403(69) 90067-8. 
588

589

$\mathbf{5 9 0}$

591

592

[34] M. A. Dietenberger, D. W. Green, D. E. Kretschmann, R. Hernandez, T. L. Highley, R. E. Ibach, J. Y. Liu, K. A. McDonald, R. B. Miller, R. C. Moody, R. M. Rowell, W. T. Simpson, L. A. Soltis, A. TenWolde, R. W. Wolfe, C. B. Vick, R. H. White, R. S. Williams, J. E. Winandy, J. A. Youngquist, Wood Handbook: Wood as an Engineering Material, Tech. Rep. FPL-GTR-113., U.S. Department of Agriculture, Forest Service, Forest Products Laboratory, Madison, Wisconsin (1999). 


\section{List of Figures}

Fig. 1. Dispersion curves for an exponentially tapered $\operatorname{rod}\left(E=10 \times(1+0.005 \mathrm{j}) \mathrm{GPa}, \rho=700 \mathrm{~kg} / \mathrm{m}^{3}\right.$, $\beta=1.55, R_{0}=0.1 \mathrm{~m}$ ): (a) real part of the wavenumber; (b) attenuation.

Fig. 2. Axial wavelength in the exponentially tapered $\operatorname{rod}\left(E=10 \times(1+0.005 \mathrm{j}) \mathrm{GPa}, \rho=700 \mathrm{~kg} / \mathrm{m}^{3}\right.$, $\beta=1.55, R_{0}=0.1 \mathrm{~m}$ ) against frequency; lines corresponding to certain number of wavelengths within the length of the rod are also marked.

Fig. 3. Experimental setup for axial FRF measurement: (a) schematic diagram; (b) accelerometers arrangement (planar view of the large face)

Fig. 4. A photograph of the experimental setup.

Fig. 5. Comparison between the experimental and the analytical results: magnitude (a) and phase (c) of the point accelerance; magnitude (b) and phase (d) of the transfer accelerance.

Fig. 6. Axial wavenumber estimated from the experimental data (using the ring of four sensors at each location): (a) real part; (b) attenuation.

Fig. 7. Axial wavenumber estimated from the experimental data - the effect of insufficient sensor spacing (here: $0.15 \mathrm{~m}$ ): (a) real part; (b) attenuation.

Fig. 8. Piecewise uniform model - schematic representation of the wave description.

Fig. 9. Shaker arrangement for flexural waves experiments.

Fig. 10. Comparison between the experimental and the numerical results: magnitude (a) and phase (c) of the point accelerance; magnitude (b) and phase (d) of the transfer accelerance.

Fig. 11. Cross-sectional view of the measured response (ODS) at $2430 \mathrm{kHz}$ dominated by the ovalling wave near the forcing location; dots represent the position of accelerometers and the arrows anchored at the dots - the displacement.

Fig. 12. Flexural wavelength distribution along the beam at different frequencies with the main adopted spacing and its double marked.

Fig. 13. Flexural wavenumber estimated from the experimental and numerical data - five FRFs spaced at $0.075 \mathrm{~m}$ with the central sensor at $0.4 \mathrm{~m}$. From experimental FRFs: (a) real part; (c) attenuation. From numerical FRFs: (b) real part; (d) attenuation.

Fig. 14. Flexural wavenumber estimated from the experimental and numerical data - five FRFs spaced at $0.075 \mathrm{~m}$ with the central sensor at $1.05 \mathrm{~m}$. From experimental FRFs: (a) real part; (c) attenuation. From numerical FRFs: (b) real part; (d) attenuation. 
623 Fig. 15. Flexural wavenumber estimated from the experimental and numerical data - five FRFs spaced 624 at $0.075 \mathrm{~m}$ with the central sensor at $1.725 \mathrm{~m}$. From experimental FRFs: (a) real part; (c) attenuation. 625 From numerical FRFs: (b) real part; (d) attenuation.

626 Fig. 16. Flexural wavenumber estimated from the experimental and numerical data - five FRFs spaced ${ }_{627}$ at $0.04 \mathrm{~m}$ with the central sensor at $1.725 \mathrm{~m}$. From experimental FRFs: (a) real part; (c) attenuation. ${ }_{628}$ From numerical FRFs: (b) real part; (d) attenuation.

629 Fig. B.1. Wave amplitudes and matrices required to fully describe the motion in a wave element. 